Article

\title{
An Improved Analytical Model for Crosstalk of SiC MOSFET in a Bridge-Arm Configuration
}

\author{
Dakang Yuan * ${ }^{\mathbb{C}}$, Yiming Zhang and Xuhong Wang * \\ Electromagnetic Theory and New Technology Laboratory, Faculty of Information Technology, \\ Beijing University of Technology, Beijing 100124, China; ymzhang@bjut.edu.cn \\ * Correspondence: yuan_dakang@163.com (D.Y.); wangxuhong8787@163.com (X.W.); \\ Tel.: +86-152-1071-8500 (D.Y.)
}

check for updates

Citation: Yuan, D.; Zhang, Y.; Wang, $X$. An Improved Analytical Model for Crosstalk of SiC MOSFET in a Bridge-Arm Configuration. Energies 2021, 14, 683. https://doi.org/ $10.3390 /$ en14030683

Received: 29 December 2020

Accepted: 26 January 2021

Published: 28 January 2021

Publisher's Note: MDPI stays neutral with regard to jurisdictional claims in published maps and institutional affiliations.

Copyright: (c) 2021 by the authors. Licensee MDPI, Basel, Switzerland. This article is an open access article distributed under the terms and conditions of the Creative Commons Attribution (CC BY) license (https:/ / creativecommons.org/licenses/by/ $4.0 /)$.
Abstract: SiC MOSFETs have an excellent characteristic of high switching speed, which can improve the efficiency and power density of converters significantly. However, the fast switching processes of SiC MOSFETs cause serious crosstalk problems in bridge-arm configurations, which restricts the devices' performances. This paper presents a detailed and accurate improved crosstalk analytical model, which takes into account the nonlinear capacitances, the parasitic inductances, the reverse recovery characteristics of the anti-parallel diodes, and the nonlinear voltage switching and damping oscillation process. The novelty of the proposed model lies in the fact that under the condition of comprehensively considering all these non-ideal factors of the bridge-arm, the effects of multiparasitic elements and multi-variables coupling to the crosstalk are hierarchically divided. The parasitic elements and their correlations are described in detail and the direct and indirect variables' impacts are clearly traced. Thus, according to the different variables switching stages, the influence processes of these parasitic elements and variables can be integrated and a complete equivalent analytical model of the crosstalk process can be derived. The simulation and experiment platforms are established and a series of experimental verifications and comparisons prove that the model can replicate experimental measurements of crosstalk with good accuracy and detail.

Keywords: analytical model; crosstalk; SiC MOSFETs; bridge-arm

\section{Introduction}

With the advantages of low switching loss, high block-voltage, high switching speed, and high thermal conductivity, SiC MOSFETs increasingly replace traditional silicon-based MOSFETs in high frequency, high efficiency, and high power density applications [1-4]. However, many kinds of converter topologies are designed based on the bridge-arm structure, the rapid change current and voltage seriously impact the parasitic elements and coupling affect the upper and lower devices, which interfere with the driver loop states and cause wrongly triggered. That is commonly referred to as crosstalk problems [5-7]. Especially in the case of SiC MOSFETs are employed in bridge-arm with high frequency, the high current and voltage switching rates significantly increase the amplitude of crosstalk [8]. Furthermore, the gate-source voltage of $\mathrm{SiC}$ MOSFETs have lower turn-on threshold voltage and negative breakdown voltage compared with silicon-based MOSFETs [9], and the gate-source parasitic capacitance is relatively smaller [10], these characteristics make the driver loop of SiC MOSFETs more susceptible to the coupling effects. These two factors contradict each other that restrict the SiC MOSFETs' performances in the bridge-arm, increase the switching loss, and even may cause unintentional turn-on to affect the stability and reliability. Moreover, the crosstalk problem not only exists in common hard-switching conditions but also occurs in the soft-switching bridge-arm [11]. Thus, in order to take full advantage of SiC MOSFETs and avoid the obstacles, crosstalk is a key factor that must be evaluated. 
Crosstalk is a phenomenon that involves many parasitic elements of the power loop and driver loop of the bridge-arm and is impacted by different variables during the switching processes. So far, the crosstalk phenomenon has been studied in many works. The most convenient and widely used model is to calculate the peak value of crosstalk, as described in [12-17]. It is ascribed to a function of the drain-source voltage switching rate, the input capacitance of the device, and the resistance of the driver loop, as expressed as follows:

$$
V_{g s}=R_{g} C_{g d} \frac{d V_{d s}}{d t}\left(1-e^{\frac{-t}{R_{g}\left(C_{g d}+C_{g s}\right)}}\right)
$$

Furthermore, in many practical engineering applications, the drain-source voltage switching rates are commonly considered as infinite to obtain the extremum of crosstalk [15-17]. The theoretical extremum of crosstalk can be derived from Equation (1) as $V_{g S_{\text {_max }}}=V_{D D} C_{g d} /\left(C_{g d}+C_{g s}\right)$, where $V_{D D}$ is the drain-source voltage when the SiC MOSFET fully turn-off. In fact, these models have undergone a series of simplifications, only the impacts of driving resistances and constant capacitances of the devices are considered, while the parasitic inductances of the bridge-arm are all ignored, which leads the accuracy cannot be fully guaranteed. More detailed models take into account the influence of other parameters of the bridge-arm, the models in Ref. [18] and Ref. [19] consider the influences of parasitic inductances, which indicate that the switching current of the bridge-arm generates an induced voltage on the common source parasitic inductance, it is superimposed on the driver loop and affects the crosstalk. Literature [20] established a detailed crosstalk equivalent analytical model that includes all the parasitic inductances of the power loop and the driver loop. The time-domain response of the crosstalk can be obtained, which is summarized as a fourth-order differential equation. However, the model still relies on the given drain-source voltage switching rate as the input source, it is not been further evaluated in detail. Moreover, since the existence of the parasitic inductances in the whole bridge-arm, the reverse recovery current of the anti-parallel diode during the turn-on process also impacts the crosstalk voltage [21,22], and in Ref. [23], the effects of the reverse recovery current on crosstalk are analyzed in detail. More and more complete considerations of the parasitic elements improve the accuracy of the crosstalk analysis but greatly increases the complexity of the model establishment. In additon, the current studies mainly focus on the coupling relationship between the crosstalk and the parasitic elements, while the analysis of the variable responses as the influence sources, caused by the bridge-arm switching processes, to the crosstalk is relatively simple.

Another critical aspect is the precise description of parasitic elements and the correlative variables, it is ultimately related to the accuracy of the crosstalk response. It is well known that the interelectrode capacitances of SiC MOSFETs have nonlinear characteristics, which should be accurately described during the voltage switching processes [24]. Although the effect of nonlinear capacitances on the switching processes of SiC MOSFETs has been thoroughly studied [21,25-28], its impacts have been not enough detailed analysis for the crosstalk, which include the effects on the switching speed of the active-controlled device and the influence on the characteristics of crosstalk response circuits. The variable responses of the bridge-arm are commonly the main influence sources of the crosstalk. Elbanhawy et al. proposed a method that the voltage switching rate is regarded as a constant value [15-17], it could cause calculation errors for crosstalk from the source. In fact, the voltage switching is a nonlinear change process [20], Ref. [20] proposes a method of using experimental measurement switching rates to facilitate the calculation accuracy, but it cannot play a role in predicting the crosstalk fundamentally and explain how the nonlinear voltage switching rate influences the response. During the whole switching process of the bridge-arm, the main variables are switched by stages (voltage switching stage, current switching stage, and the reverse recovery process of the anti-parallel diode). In order to get the entire crosstalk response, the coupling effects of these variables and the coupling parasitic elements are different, which should be discussed separately and precisely [21,24]. In addition, some variable responses have overlapping situations, such 
as the reverse recovery current drop process coincides with the voltage rising stage in the turn-on process [8,29], which increases the complexity of the analysis. Moreover, due to the inertial effect of the parasitic elements, there are oscillation responses of the voltage and current after the full switching process of the bridge-arm [30-33], which are also coupled to the driver loop and impact the crosstalk. To sum up, although these special characteristics and response processes greatly cumber the model design, they ultimately related to the accuracy of the crosstalk response. However, there is no complete model that can describe the crosstalk process correlates with these parasitic elements and the key variable responses of the bridge-arm particularly and comprehensively.

This paper presents a detailed and accurate analytical model of crosstalk for SiC MOSFETs applied in the bridge-arm configuration. In view of the complex coupling characteristics of multi-parasitic elements and multi-variables in the crosstalk response process, the influencing factors are summarized first. Crucially, the nonlinear characteristics of the interelectrode capacitances, the reverse recovery characteristics of the anti-parallel diodes, the parasitic inductances of the power loop, and the nonlinear voltage switching and damping oscillation process are analyzed in detail to ensure accuracy from the source. According to the switching processes of the bridge-arm, the correlation of the variables and parasitic elements with crosstalk in each stage are distinguished and the influence mechanisms behind these parameters are traced clearly. On this basis, a detailed analytical model of crosstalk is established, the source impact variables and the critical parasitic elements are all considered and combined, their direct or indirect coupling effects to the crosstalk in different switching stages are clarified. The equivalent circuits of the switching stages are established and calculation procedures are derived hierarchically. In the most critical voltage switching stages, the multitude of impact factors can be linked to the response of the drain current, it plays a key role in the impacts of crosstalk from the power loop to the driver loop, and then the crosstalk can be further evaluated with this unique direct impact source. A double pulse test platform composed of $600 \mathrm{~V} / 36 \mathrm{~A} \mathrm{SiC}$ MOSFETs is set up and a series of experimental results are provided to verify the correctness and accuracy of the proposed analytical model. Further, the simulation calculations and experimental results are compared with the calculation results in $[17,24]$, and the theoretical extremums, the results show that the proposed model can describe crosstalk response in more detail and accuracy.

\section{Analysis of Crosstalk Response Processes and Related Factors}

A double-pulse test based on a bridge-arm configuration is a simple and effective way to analyze the crosstalk process. In this paper, a double-pulse test circuit is established to explain the crosstalk phenomenon. The equivalent circuit model is shown in Figure 1. The DC input bus voltage $V_{D D}$ is modeled as a constant voltage source, and the output current $I_{L O A D}$ is set as a constant current source. The upper SiC MOSFET, $Q_{H}$, is the active-controlled device, which works in the normal turn-on/off states, the lower $\mathrm{SiC}$ MOSFET $Q_{L}$ is set at a constant turn-off state, which is used to observe the $V_{g s L}$ crosstalk response. During the $Q_{H}$ cut-off period, the load current $I_{L O A D}$ flows through the antiparallel freewheeling diode of $Q_{L}, D_{b L}$. 


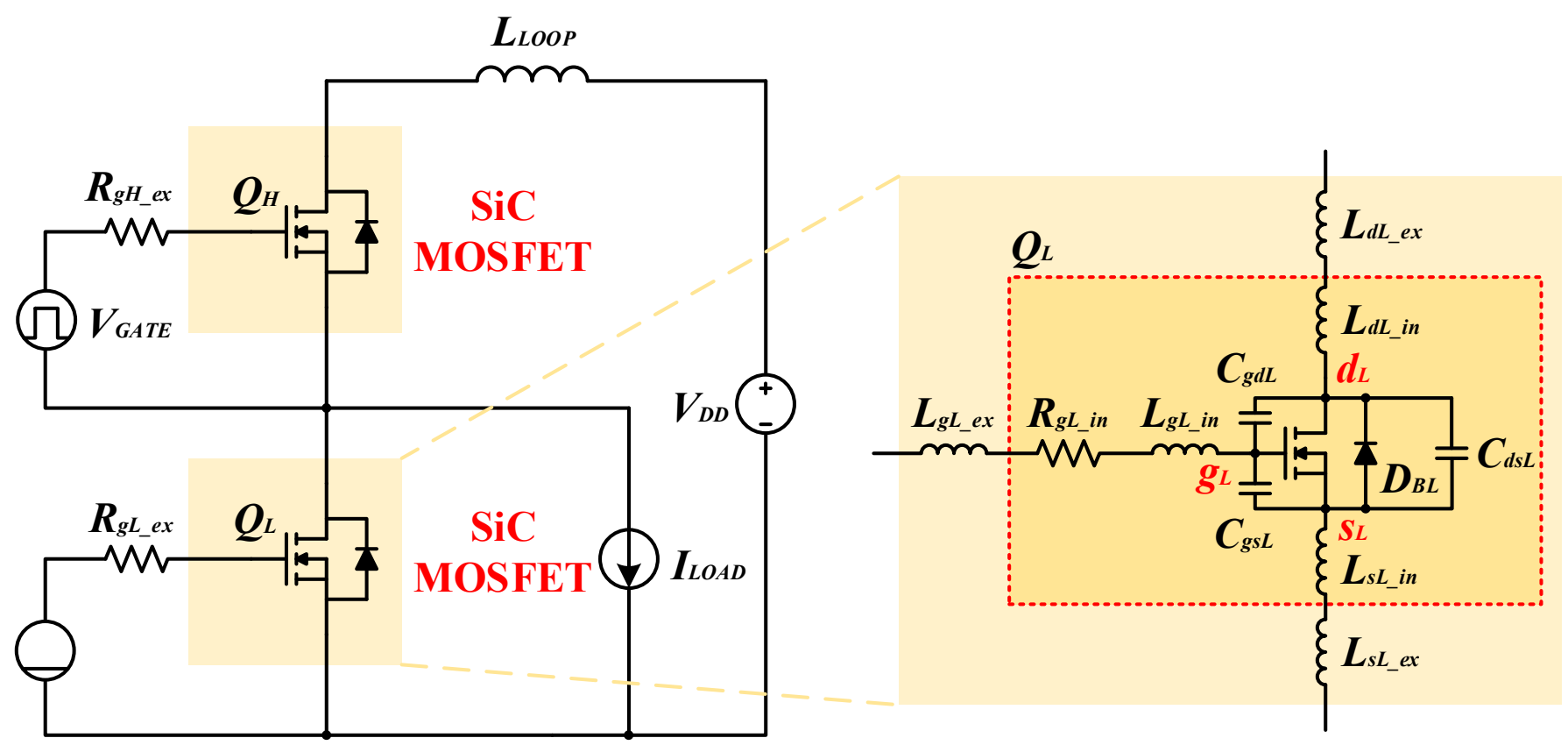

Figure 1. Equivalent model of double pulse test circuit and the parasitic elements of SiC MOSFET and bridge arm.

The parasitic elements and of SiC MOSFETs devices and the bridge-arm circuits should be considered as comprehensively as possible to ensure accuracy. In practice, there are usually parasitic inductances distributed in the power loop, which are summarized as $L_{L O O P}$. The parasitic elements of the SiC MOSFETs need to consider the internal of the device package and the external circuit traces, as shown in Figure 1. Taking $Q_{L}$ as an example, the internal parasitic elements include interelectrode capacitances $C_{g s L}, C_{g d L}$, $C_{d s L}$, where they have nonlinear characteristics, parasitic inductances of gate, drain, and source, $L_{g L_{-} i n}, L_{s L_{-} i n}$, and $L_{d L_{-} i n}$, internal gate driver resistance $R_{g L_{-} i n}$, and the antiparallel freewheeling diode $D_{B L}$, where it has reverse recovery characteristic. The external parasitic elements are the stray inductances of the electrode pins and the circuit traces connected with the gate, source, and drain, which are represented by $L_{g H \_} e x, L_{d H_{-} e x}$, and $L_{s H \_e x}$, respectively. Generally, there is an external driver resistance $R_{g L_{\_} e x}$ in the driver loop to regulate the switching speed. In order to facilitate the analysis, denoting $L_{g H}=L_{g H} e x+L_{g H} i n, L_{d H}=$ $L_{d H_{-} e x}+L_{d H_{-} i n}, L_{s H}=L_{s H_{-} e x}+L_{s H_{-} i n}$ as the total gate, drain, and source inductances of $Q_{L}$, and $R_{g L}=R_{g H_{-} e x}+R_{g H_{-} i n}$ as the total gate resistance of $Q_{L} \cdot Q_{H}$ has the same structure.

At present, most discrete $\mathrm{SiC}$ MOSFET commercial products have two package structures, non-Kelvin package and Kelvin package. For non-Kelvin package device (such as TO-247-3), the parasitic inductance of the source should be emphasized, it is contained in the power loop and the driver loop, which makes the driver loop more vulnerable to the coupling effect of the current variation of the power loop, as is shown in Figure 2a. While the source of the Kelvin package device (such as TO-247-4) is separated for the driver loop and the power loop, which could get rid of this drawback, as shown in Figure 2b. However, the internal common source parasitic inductance cannot be completely ignored, the coupling impacts from the power loop still exist [24]. Moreover, non-Kelvin package $\mathrm{SiC}$ MOSFETs are widely adopted in engineering applications. Thus, the crosstalk with non-Kelvin package SiC MOSFETs is mainly discussed in this paper. The impacts of common source parasitic inductance are greater than that of the Kelvin package devices, and the analysis processes are more complicated due to the superposition influences of the power loop. This analysis method can also be simplified and transplanted to similar Kelvin packaged discrete devices and modules. 


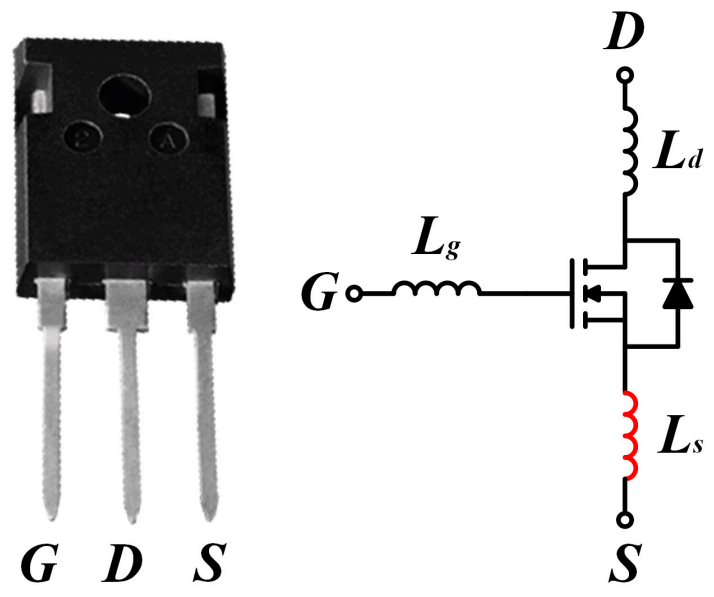

TO-247-3

(a)

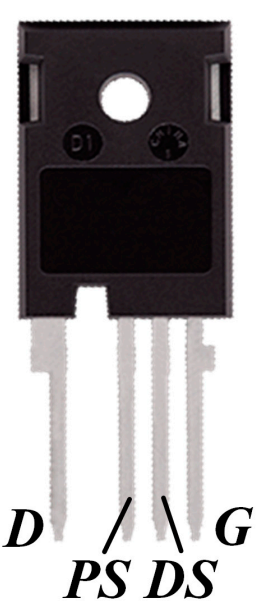

TO-247-4

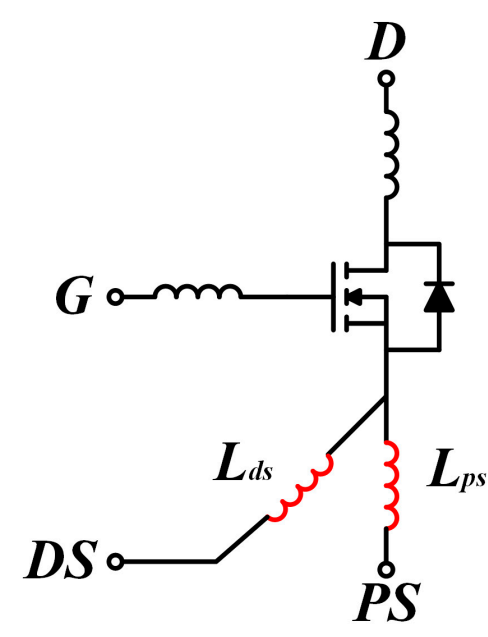

(b)

Figure 2. Structures of discrete SiC MOSFET with non-Kelvin package and Kelvin package. (a) non-Kelvin package (TO-247-3). (b) Kelvin package (TO-247-4).

In the bridge-arm configuration, the parasitic elements and their characteristics not only directly determine the response characteristics of the crosstalk voltage, but also affects the voltage and current switching process of the bridge-arm, which indirectly determine the impact sources to the crosstalk. Therefore, all these parasitic elements and their influence on the whole switching process should be integrally considered. At present, the operation characteristics and switching processes modeling of various types of power devices have been thoroughly studied in many researches. For a non-linear variation switching process of the device, the piecewise linear model is the most convenient and widely used analytical model [34-36]. Based on the piecewise linear analytical model for the switching devices, the analysis can be further related to the influence on the switching processes and crosstalk responses of bridge-arm configuration. From the perspective of crosstalk voltage, the key parameters and variables associated with crosstalk in each stage are further extracted. Thus, a thorough analysis of the influences of parasitic elements and switching variables on crosstalk and switching variables is presented. Figure 3 illustrates a typical double-pulse test turn-on and turn-off qualitative switching waveforms. Among them, Stage 2 and Stage 7 are the current switching stages of the turn-on and turn-off process, respectively. Stage 3 and Stage 6 are the voltage switching stages of the turn-on and turn-off process, respectively. The fluctuation of $V_{g s L}$ is displayed in red in Figure 3. 


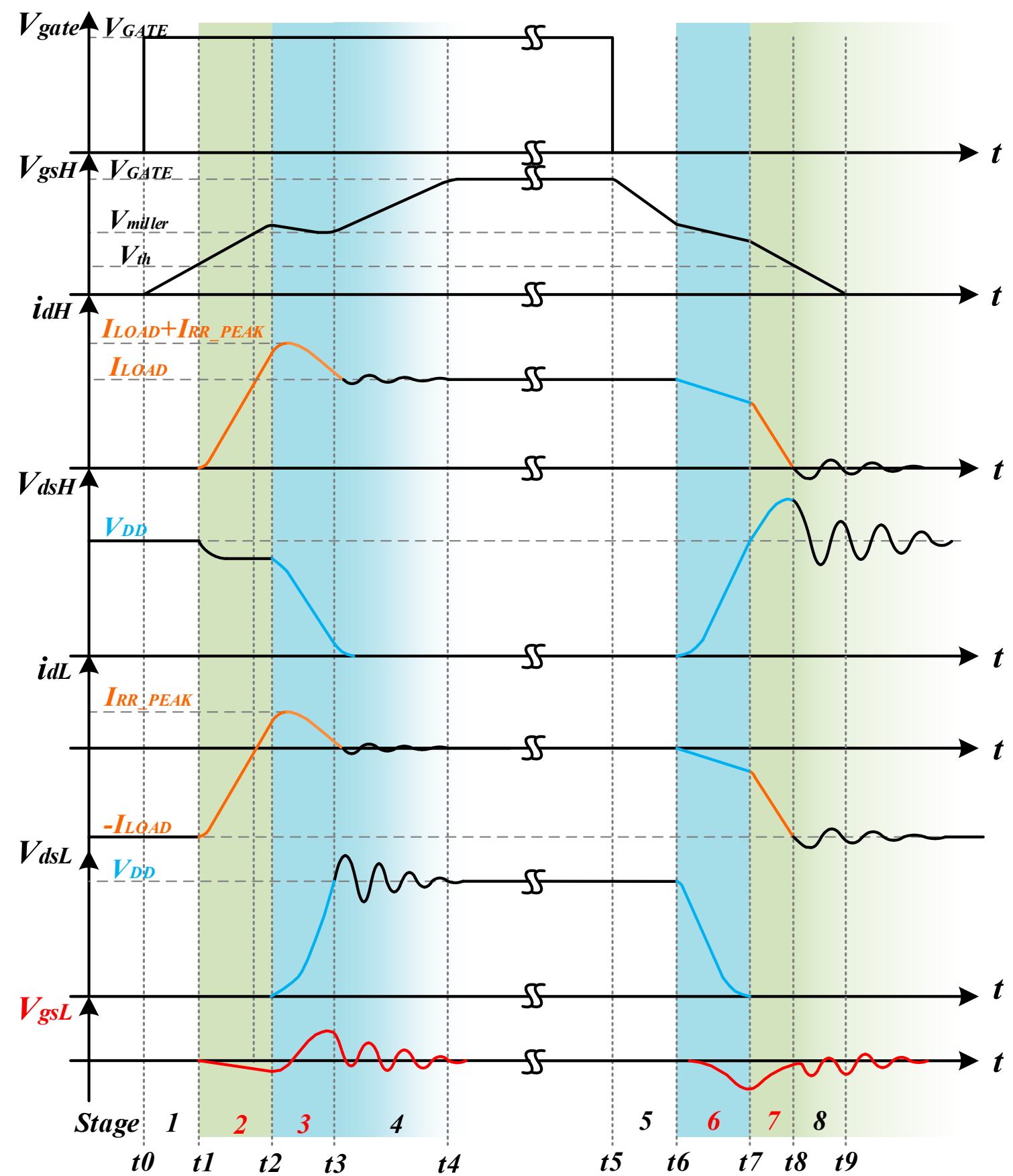

Figure 3. Qualitative analytical switching sequence of turn-on and turn-off stages.

The current and voltage switching in the power loop of the bridge-arm cause two key effects that affect the crosstalk. The first one is the rapid current switching generates an induced voltage on the common source parasitic inductance, $L_{S L}$, which is superimposed in the driver loop. The second one is the voltage switching causes a displacement current of the gate-drain capacitance, $C_{g d L}$, which forms a circuit through the driver loop. Both of them are directly related factors to the responses of $V_{g s L}$.

More specifically, in the turn-on process of $Q_{H}$, the supply current of $I_{L O A D}$ transfers from $D_{B L}$ to the channel of $Q_{H}$ first, which corresponds to Stage 2 in Figure 3. The freewheeling current flowing through $D_{B L}$ decreases rapidly. Due to the reverse recovery characteristics, after the freewheeling current drops to zero, the minority carriers of $D_{B L}$ need to be elicited first, which causes a positive reverse recovery current increase. Thereafter, $D_{B L}$ turns to the reverse-biased state and the blocking voltage starts to build up. 
During the whole process before the formation of the depletion layer of $D_{B L}$, there is no reverse-blocking capability and $V_{d s L}$ does not increase. Thus, only the induced voltage of $L_{s L}$ is coupled to the driver loop of $Q_{L}$ in this stage. The equivalent circuit of this stage is shown in Figure 4a.

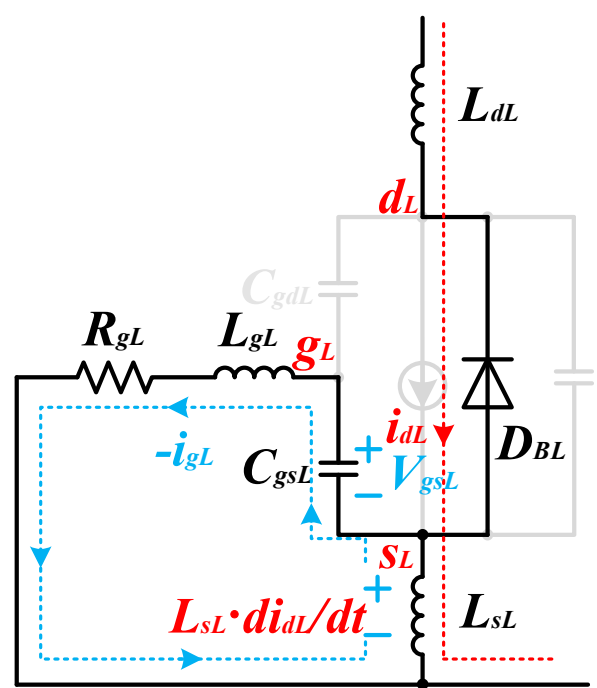

(a)

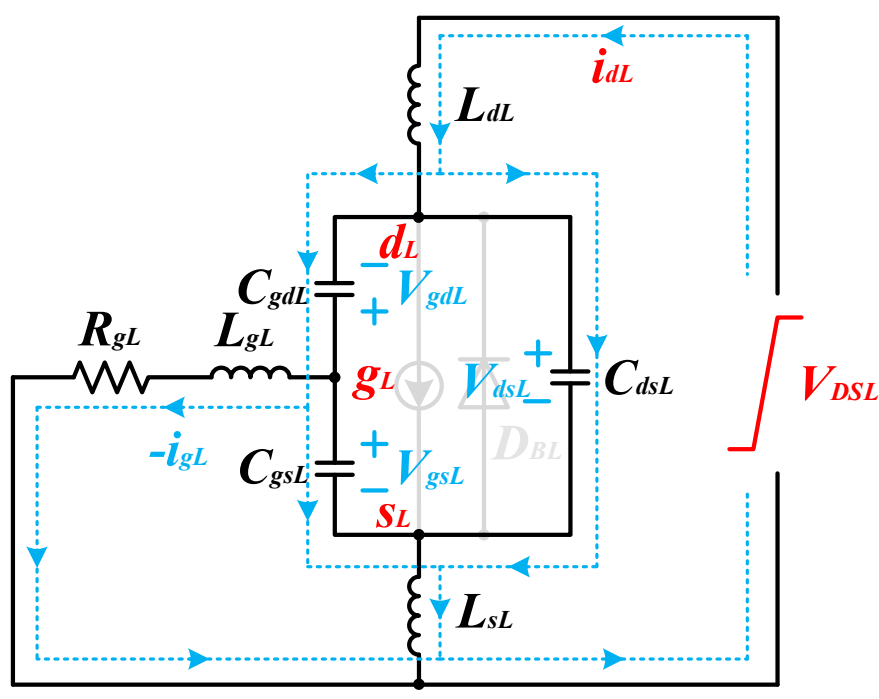

(b)

Figure 4. Equivalent circuit of $Q_{L}$ for crosstalk analysis during turn-on process. (a) current decrease stage. (b) voltage rise stage.

The equations of the driver loop of this stage can be expressed as

$$
\begin{gathered}
i_{g L}=C_{g s L} \frac{d V_{g s L}}{d t} \\
R_{g L} i_{g L}+L_{g L} \frac{d i_{g L}}{d t}+V_{g s L}=-L_{s L} \frac{d i_{d L}}{d t}
\end{gathered}
$$

During Stage 3, the drain-source voltage of $Q_{L}$ is built up (the blocking voltage of $D_{B L}$ increases). The gate-drain capacitance $C_{g d L}$ is charged, the charging current flows through $C_{g s L}$ and the driver loop of $Q_{L}$, which causes a positive crosstalk voltage of $V_{g s L}$. In addition, it must be noted that the power loop maintains the reverse recovery current at the end of the previous stage, which approaches its peak value and keeps the rising rate. It can be considered as the initial conditions during the increase process of $V_{d s L}$. With the increase of $V_{d s L}$, the current charging the parasitic capacitances of $Q_{L}\left(C_{g d L}, C_{d s L}\right.$, and $\left.C_{g s L}\right)$, $i_{d L}$, decreases gradually. The equivalent circuit of this stage is shown in Figure $4 \mathrm{~b}$. The induced voltage on $L_{s L}$ becomes negative, which also causes a positive impact on $V_{g s L}$. The circuit equations are established as

$$
\begin{gathered}
V_{g s L}=V_{g d L}+V_{d s L} \\
i_{d L}=-C_{g d L} \frac{d V_{g d L}}{d t}+C_{d s L} \frac{d V_{d s L}}{d t} \\
i_{g L}=C_{g d L} \frac{d V_{g d L}}{d t}+C_{g s L} \frac{d V_{g s L}}{d t} \\
V_{D S L}=L_{d L} \frac{d i_{d L}}{d t}-V_{g d L}-L_{g L} \frac{d i_{g L}}{d t}-R_{g L} i_{g L} \\
V_{D S L}=\left(L_{d L}+L_{s L}\right) \frac{d i_{d L}}{d t}+V_{d s L}+L_{s L} \frac{d i_{g L}}{d t}
\end{gathered}
$$


where $V_{D S L}$ is the voltage between the phase node and ground.

When $V_{d s H}$ reaches the on-state voltage, $Q_{H}$ is completely cut-off. $V_{d s L}$ rises to $V_{D D}$ and keeps the rising rate at $t 4$, then it starts oscillating due to the parasitic inductances of the power loop and the parasitic capacitances of $Q_{L}$. The oscillations of voltage and current are coupled to the driver loop through $C_{g d L}$ and $L_{s L}$, which further cause fluctuations on crosstalk, as shown waveforms after $t 3$ in Figure 3. The equivalent circuit is the same as Figure $4 \mathrm{~b}$.

In the turn-off process of $Q_{H}$, its channel resistance starts to increase first, which corresponds to Stage 6 in Figure 3. $V_{d s H}$ starts to rise and $V_{d s L}$ decreases, $D_{B L}$ withstands a negative voltage and the current does not yet switch in this stage. However, the charging and discharging of the parasitic capacitances lead part of the bridge-arm current transferred to maintain the constant of the load current. The initial states of the parasitic capacitances discharge current of $Q_{L}$ are zero, and the equivalent circuit is shown in Figure 5a. Same as Stage 3 in the turn-on process, the voltage and current variations all affect the crosstalk response, but the variables' directions are opposite. $V_{g s L}$ has a negative fluctuation. When $V_{d s L}$ drops to the forward turn-on voltage of $D_{B L}$, the voltage switching is completed and the current begins to divert from the channel of $Q_{H}$ to $D_{B L}$. A negative induced voltage on $L_{S L}$ is superimposed on the diver loop of $Q_{L}$, which causes a positive fluctuation impact on $V_{g s L}$. as shown the Stage 7 in Figure 3. The equivalent circuit is shown in Figure 5b. the expressions of these two stages are the same as the turn-on stages, Stage 3 and Stage 2, respectively.

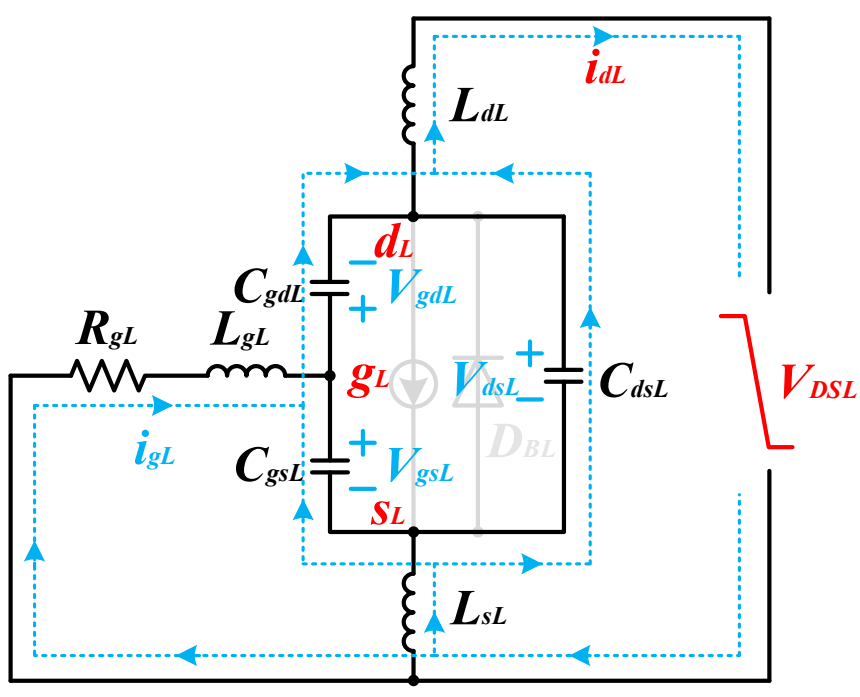

(a)

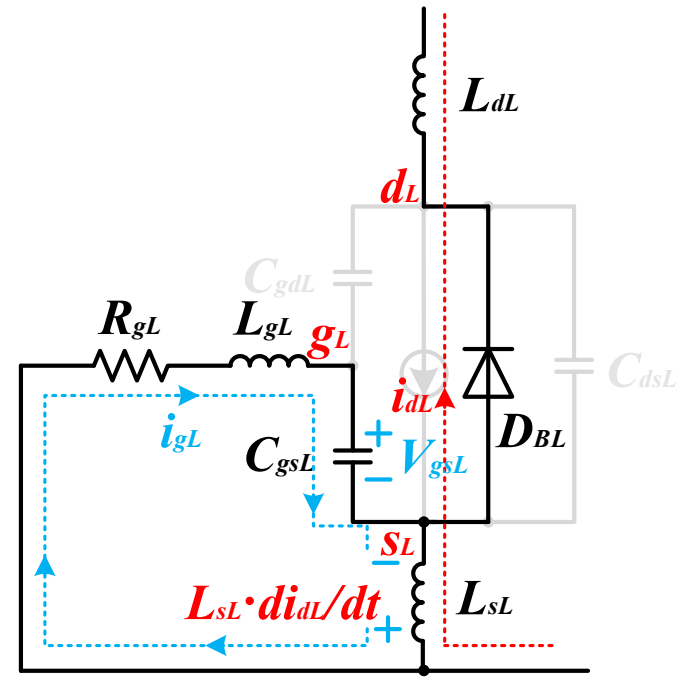

(b)

Figure 5. Equivalent circuit of $Q_{L}$ for crosstalk analysis during turn-off process. (a) voltage drop stage. (b) current rise stage.

Based on the analyses above, it can be seen that the response of $V_{g s L}$ in different stages corresponds to different variables of the bridge-arm and correlate with many parasitic elements, which makes the modeling cumbersome. Especially in the voltage switching processes, the coupling of the two influence paths associates five independent state variables, the crosstalk response of $V_{g s L}$ is simplified to a fourth-order differential equation, it is difficult to derive the time domain solutions. Moreover, compared with Equation (4) in [24], the order of $V_{g s L}$ response is significantly increased, which is due to the influence of parasitic inductances are taken into account.

Further, there are several special parameter characteristics of SiC MOSFETs in the above-mentioned switching processes also affect the accuracy of crosstalk that must be carefully considered. SiC MOSFETs have smaller parasitic capacitances than silicon-based devices, and their values are nonlinear versus the drain-source voltage. For the SiC 
MOSFETs with type CMD080120D, the nonlinear curves are shown in Figure 6. It can be found that especially the nonlinear variation of reverse capacitance $C_{r s S}\left(C_{g d}\right)$, it decreases nearly a hundredfold with the increase of $V_{d s L}$. This variation has an obvious influence on the characteristic of the $C_{g d}$ displacement current loop, which directly associates with the crosstalk response according to the Equations (5)-(8). Literature [24] also clearly verified the impact of the nonlinear characteristics of $C_{g d L}$ on the accuracy of the crosstalk response.

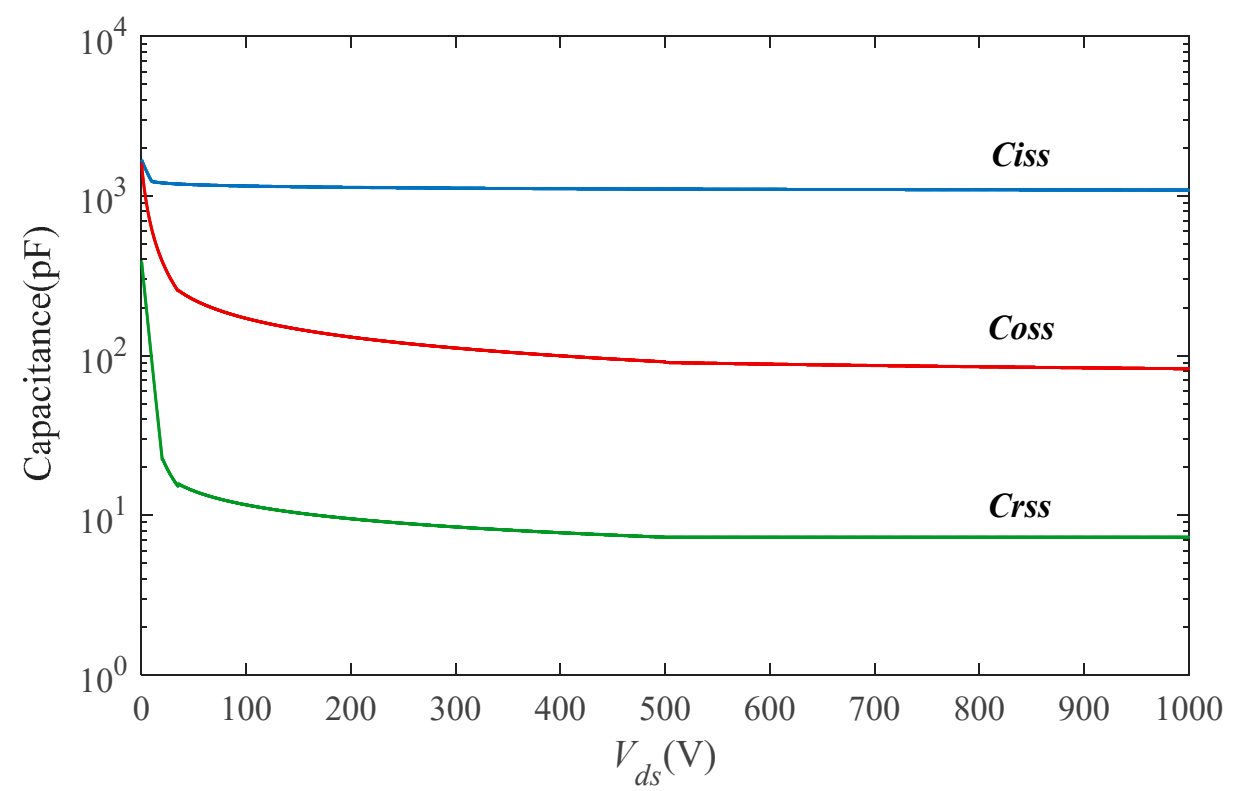

Figure 6. Parasitic capacitances in relationship with the drain-source voltage.

Moreover, the nonlinear variation of the parasitic capacitances of $Q_{H}$ also affects the voltage switching rate. According to the literature [20,24,37], the switching rate of $V_{d s H}$ is determined by the driving current $i_{g H}$ and the $C_{g d H}$. Due to the restriction of the voltage dynamic equilibrium of the whole bridge-arm, the response of $V_{d s L}$ presents a nonlinear variation process. As one of the input sources of the crosstalk in Stage 3 and Stage 6, the response precision of $V_{d s L}$ must be ensured. Thus, this indirect effect of nonlinear capacitances also needs to be considered.

For the response of $V_{d s L}$, another critical factor is the impact of parasitic inductances. Literature [24] indicates that the switching process of $V_{d s L}$ is nonlinear and simplified with a piecewise linear method, but it did not take into account the influence of parasitic inductances of the power loop. It considers that the response of $V_{d s L}$ is the reversed response of $V_{d s H}$, and the crosstalk amplitude of $V_{g s L}$ reaches the maximum when $V_{d s L}$ reaches the bus voltage $V_{D D}$ in the turn-on process. These settings will bring errors from the source. In practice, due to the parasitic inductances of the power loop, on the one hand, the parasitic inductances participate in the voltage switching process, which shared parts of voltage with the transient variation of $V_{d s L}$, the sum of $V_{d s H}, V_{d s L}$ and the induced voltages of parasitic inductances the is $V_{D D}$. On the other hand, $V_{d s L}$ will continue to oscillate after $V_{d s L}$ reaches $V_{D D}$ in the turn-on process, which will impact the driver loop and cause fluctuation of $V_{g s L}$. The time of $V_{g s L}$ to reach the maximum value, Tm, will also be delayed, as shown in Figure 7. 


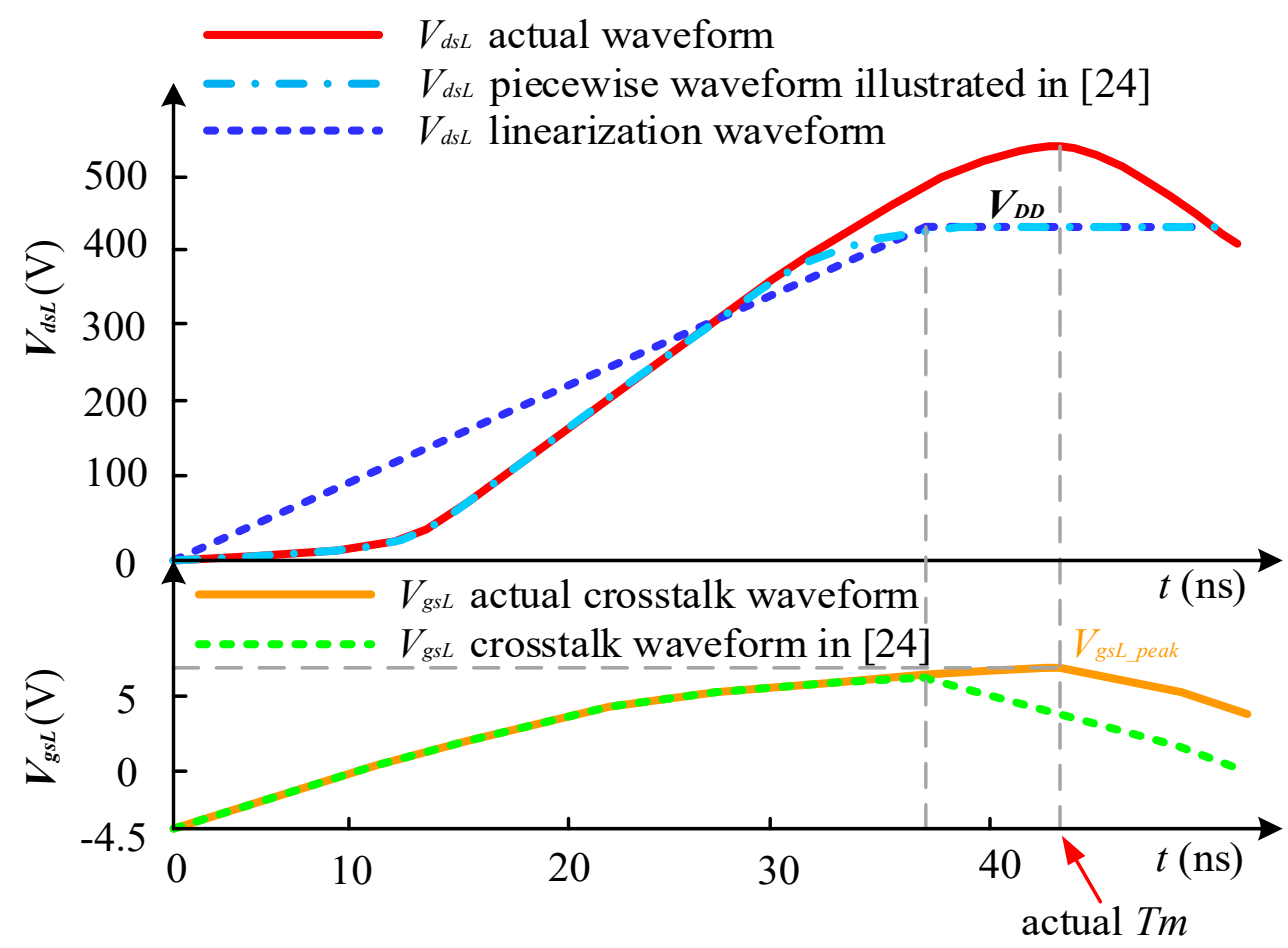

Figure 7. The formation of crosstalk in voltage switching stage of turn-on process.

To sum up, the nonlinear characteristics of parasitic capacitances, the nonlinear variation of the drain-source voltage of $Q_{H}$, the parasitic inductance of the power loop, and the voltage oscillations are all correlated to the crosstalk response, and they are coupled with each other multiply that increases the obstacles to the modeling. Therefore, a detailed and accurate model is necessary for the design guide of bridge-arm configuration to help evaluate crosstalk, as well as it could explain the influence mechanism of relevant parameters.

\section{The Analytical Model for Crosstalk}

According to the analyses in Section 2, the crosstalk phenomenon lasts almost throughout the whole switching process of the bridge-arm and different parasitic elements are coupled in different switching stages. It is necessary to consider the parasitic elements specifically and their correlative effects on the related variables in each stage should be studied deeply. Establishing accurate models of them is an important step for the crosstalk analysis. So, the modeling of special parameters and main variables are provided first, based on them, a crosstalk model can be derived in detail.

\subsection{Model Establishments of Special Parameters and Main Variables}

\subsubsection{Nonlinear Capacitances}

The device manufacturer provides the curves of the input capacitance $C_{i s s}$, the output capacitance $C_{o s s}$, and the reverse capacitance $C_{r s s}$ in the datasheet in general. According to the method provided in Refs. [26,27], the values of them can be piecewise fitted with high precision, which can be described by the equation

$$
C\left(V_{d s}\right)=C_{j 0}\left(1+\frac{V_{d s}}{V_{j}}\right)^{-m}
$$

where $C_{j 0}$ is the capacitance value when $V_{d s}=0, V_{j}$ is the built-in voltage and can be treated as an undetermined coefficient, $m$ is the capacitance gradient factor. They can be adjusted according to the range of $V_{d s}$. Figure 8 shows the fitted capacitances simulation curves compared with the datasheet curves. 


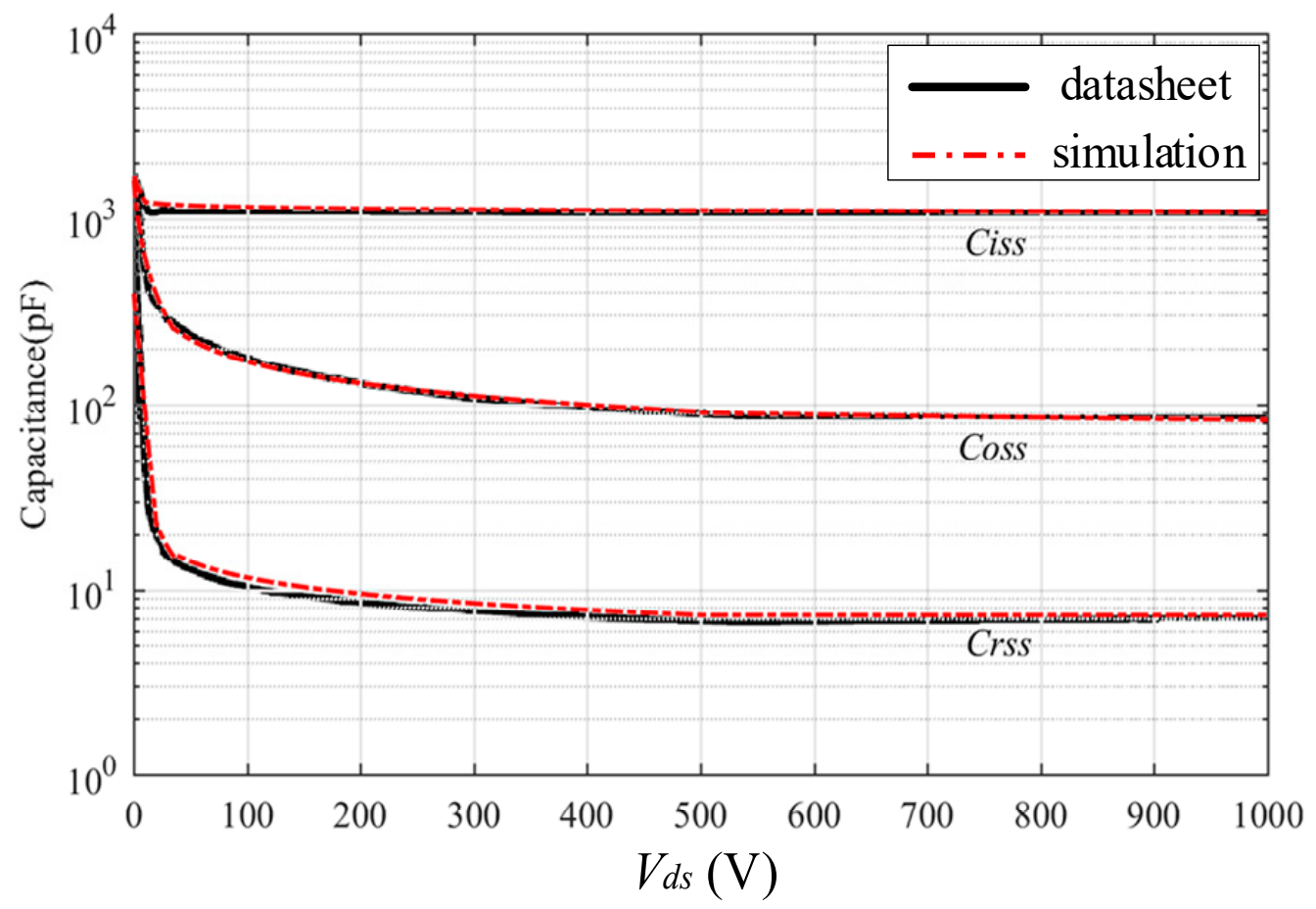

Figure 8. Comparison between the data from datasheet and the simulation.

Interelectrode capacitances are usually used in actual model establishment and calculations, which can be extracted according to Equation (10). Their comparison with the datasheet curves is shown in Figure 9. Since $C_{g s}$ and $C_{d s}$ are much larger than $C_{g d}$, the interelectrode capacitances curves are almost the same with the parasitic capacitance curves from the datasheet.

$$
\left\{\begin{array}{l}
C_{i s s}=C_{g s}+C_{g d} \\
C_{o s s}=C_{d s}+C_{g d} \\
C_{r s s}=C_{g d}
\end{array}\right.
$$

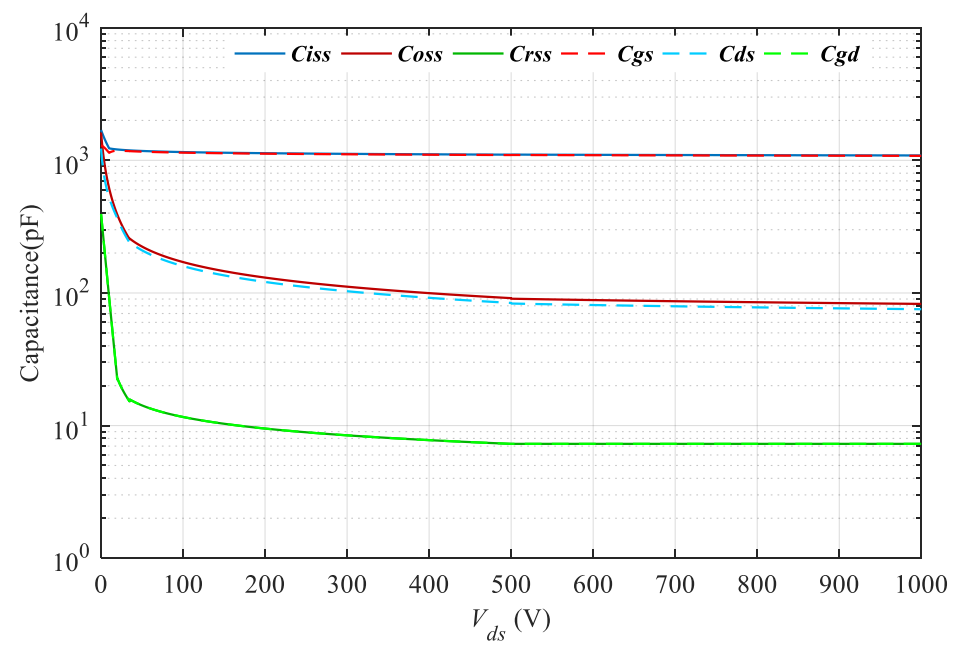

Figure 9. Comparison between the capacitances from datasheet and the interelectrode capacitances. 


\subsubsection{Current Switching Rate and Reverse Recovery Current of $D_{B L}$}

Essentially, the current switching rate is determined by the active-controlled device. When $Q_{H}$ works in the saturation region, $Q_{H}$ is modeled as a dependent current source controlled by $V_{g s H}$. The expression of the channel current is given by

$$
i_{c h}=g_{f}\left(V_{g_{s H}}-V_{t h}\right)
$$

where $i_{c h}$ is the channel current of $Q_{H}, g_{f}$ is the transconductance coefficient and it remains at the maximum. The rising rate of $V_{g s H}$ is entirely dependent on the driver loop characteristics of $Q_{H}$. It worth noting that the induced voltage on $L_{s H}$ is superimposed in the driver loop, which provides negative feedback to the driver loop [21,37]. This factor restricts the rising rate of $V_{g s H}$ and makes the current switching rate reach a maximum. The circuit equations of the $Q_{H}$ driver loop can be expressed as

$$
\begin{gathered}
i_{g H}=C_{g s H} \frac{d V_{g s H}}{d t} \\
R_{g H} i_{g H}+\left(L_{g H}+L_{s H}\right) \frac{d i_{g H}}{d t}+V_{i d s H}+V_{g s H}=V_{G A T E}
\end{gathered}
$$

where $V_{i d s H}$ is induced voltage on $L_{s H}$ caused by the changing of $i_{d H}$

$$
V_{i d s H}=L_{s H} \frac{d i_{d H}}{d t}
$$

When the current switching rate reaches the maximum, the induced voltage of the parasitic inductances of the power loop remains constant, and the interelectrode capacitances do not have charge current, thus, the current of the power loop can be considered as $i_{d H}=i_{c h}$.

In Stage 2, when the channel current rises to $I_{L O A D}$, the freewheeling current of $D_{B L}$ drops to zero and starts the reverse recovery process, $i_{d L}$ increases in a positive direction and its rate remains the same as the drop rate before, it is because the equilibrium state remains unchanged (the characteristics of the $Q_{H}$ driver loop and the transconductance). In order to obtain the rising duration of the reverse recovery current, the diode model in reference [38] is employed, which separates the reverse recovery process and charging process of junction capacitance into two stages. It is more suitable to describe the processes of current rising and voltage build-up respectively, as is shown in Figure 10. In general, the reverse recovery charge given in the datasheet is the charge for the entire reverse recovery process, $Q_{r r}$; thus, the charge of the reverse recovery process, $Q_{r s}$, needs to derive according to the provided test conditions. The equations are established as follows:

$$
\begin{gathered}
t_{r r}=t_{r s}+t_{r f} \\
Q_{r r}=Q_{r s}+Q_{r f} \\
i_{r r_{-} \text {peak }}=\left.t_{r s} \frac{d i_{d L}}{d t}\right|_{i_{d L}=I_{L O A D}} \\
Q_{r r}=\frac{1}{2} i_{r r_{-} \text {peak }} t_{r r} \\
Q_{r s}=\frac{1}{2} i_{r r_{\text {p peak }}} t_{r s}
\end{gathered}
$$




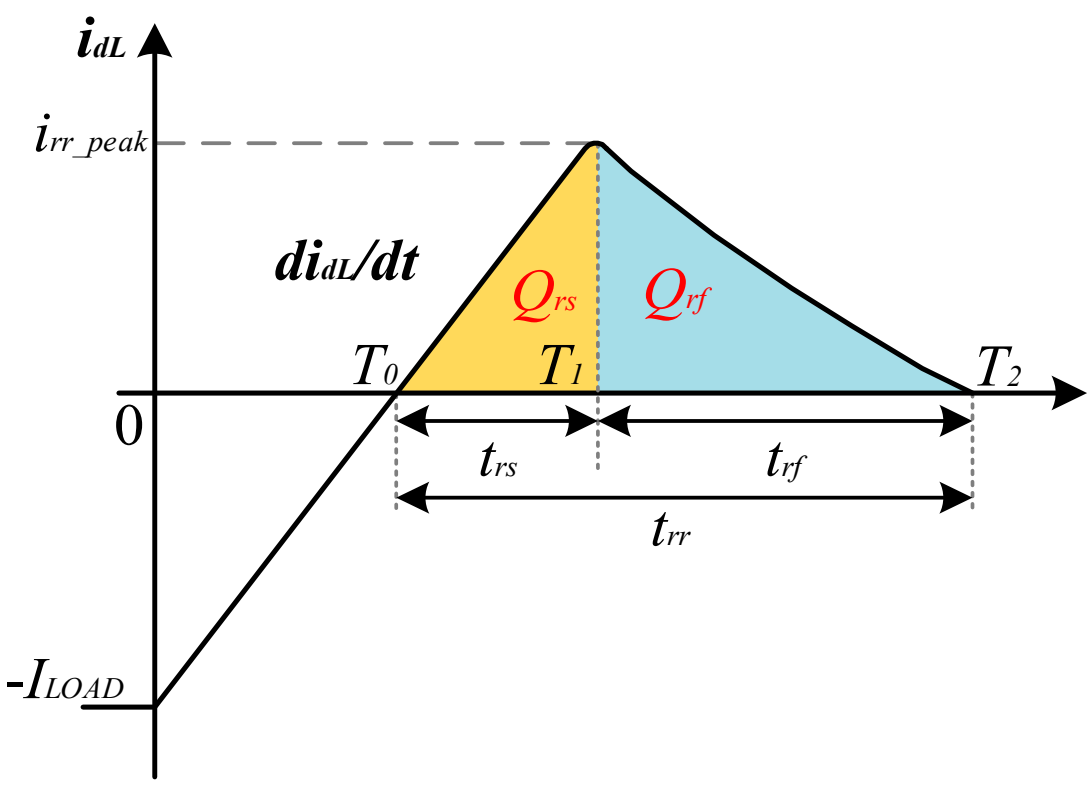

Figure 10. Modeling of the reverse recovery process of the antiparallel freewheeling diode.

The test conditions are listed in Table 1, the relationship between $Q_{r s}$ and $Q_{r r}$ can be derived as Equation (20).

$$
Q_{r s}=2\left(\frac{Q_{r r}}{t_{r r}}\right)^{2} /\left.\frac{d i_{d L}}{d t}\right|_{i_{d L}=I_{L O A D}}
$$

Table 1. Antiparallel Freewheeling Diode Characteristics and Reverse Recovery Test Parameters.

\begin{tabular}{ccc}
\hline Parameter & Value & Test Condition \\
\hline$V_{D D}(\mathrm{~V})$ & 3.3 & $V_{g s}=-5 \mathrm{~V}, I_{L O A D}=20 \mathrm{~A}, V_{D D}=800 \mathrm{~V}$ \\
$t_{r r}(\mathrm{~ns})$ & 32 & $d i_{d} / \mathrm{dt}=2400 \mathrm{~A} / \mathrm{us}$ \\
$Q_{r r}(\mathrm{nC})$ & 192 & \\
\hline
\end{tabular}

Therefore, in the analysis of the actual current switching of the bridge-arm, according to $Q_{r s}$ and the rising rate of $i_{d L}$, the reverse recovery current value at the time of $D_{B L}$ turning to the reverse-biased can be obtained, which is the initial condition of the voltage switching response in the next stage.

\subsubsection{Voltage Switching Rate}

The impact of the voltage switching is the most obvious source factor on the crosstalk. In the bridge-arm configuration, the voltage switching is controlled by the active-controlled device. When the drain-source voltage of the active-controlled device changes, the drainsource voltage of the passive device changes accordingly to keep the bus supply voltage constant.

For the active-controlled $\mathrm{SiC}$ MOSFET, $Q_{H}$, the current discharges $C_{g d}$ in this stage, meanwhile, $V_{g s}$ is clamped to the $V_{\text {miller }}$ and keeps constant, which has been well known as miller platform voltage. the gate driver works like a current source. There is not a significant charging current through $C_{g s H}$. $i_{g}$ can be express as

$$
i_{g H}=C_{g d H} \frac{d V_{g d H}}{d t}=C_{g d H} \frac{d\left(V_{\text {miller }}-V_{d s H}\right)}{d t}=-C_{g d H} \frac{d V_{d s H}}{d t}
$$

$V_{g s H}$ keeps at $V_{\text {miller, }}$, which has a clamping effect on the driver loop, the maximum current that the driver loop can reach is limited. Therefore, $i_{g H}$ remains constant, and the 
induced voltage on the parasitic inductances of the driver loop can be ignored. The driver loop equation of $Q_{H}$ can be expressed as

$$
V_{G A T E}=R_{g H} i_{g H}+V_{\text {miller }}+V_{i d s H}
$$

where $V_{i d s H}$ is induced voltage on $L_{s H}$ caused by $i_{d H}$. Combining (14), (21), and (22), the switching rate of $V_{d s H}$ can be obtained as

$$
\frac{d V_{d s H}}{d t}=-\frac{V_{G A T E}-V_{\text {miller }}}{R_{g H} C_{g d H}}+\frac{L_{s H}}{R_{g H} C_{g d H}} \frac{d i_{d H}}{d t}
$$

It can be seen from (23) that the nonlinear variation of $V_{d s H}$ is affected by the nonlinear characteristics of $C_{g d H}$ and the rate of $i_{d H}$. In addition, the common source parasitic inductance $L_{s H}$ provides negative feedback to the switching rate of $V_{d s H}$. In the turn-on process, since the reverse recovery current decrease, a negative induced voltage is generated on $L_{s H}$, which will increase the drop rate of $V_{d s H}$. Conversely, it suppresses the rising rate of $V_{d s H}$ in the turn-off process.

Due to the parasitic inductances exist in the power loop, the response of $V_{d s L}$ cannot be directly described as a reversed response of $V_{d s H}$. In this paper, $V_{d s L}$ is equivalent to a response of the whole parasitic capacitances of $Q_{L}$ charged by the applied voltage $V_{D D}-V_{d s H}$, where all the parasitic inductances of the power loop and the driver loop branch are taken into consideration. The equivalent circuits are shown in Figure 11. In this way, an accurate nonlinear input is given from the source and the influences of parasitic elements are thoroughly considered. These settings can ensure the accuracy of the response of $V_{d s L}$.

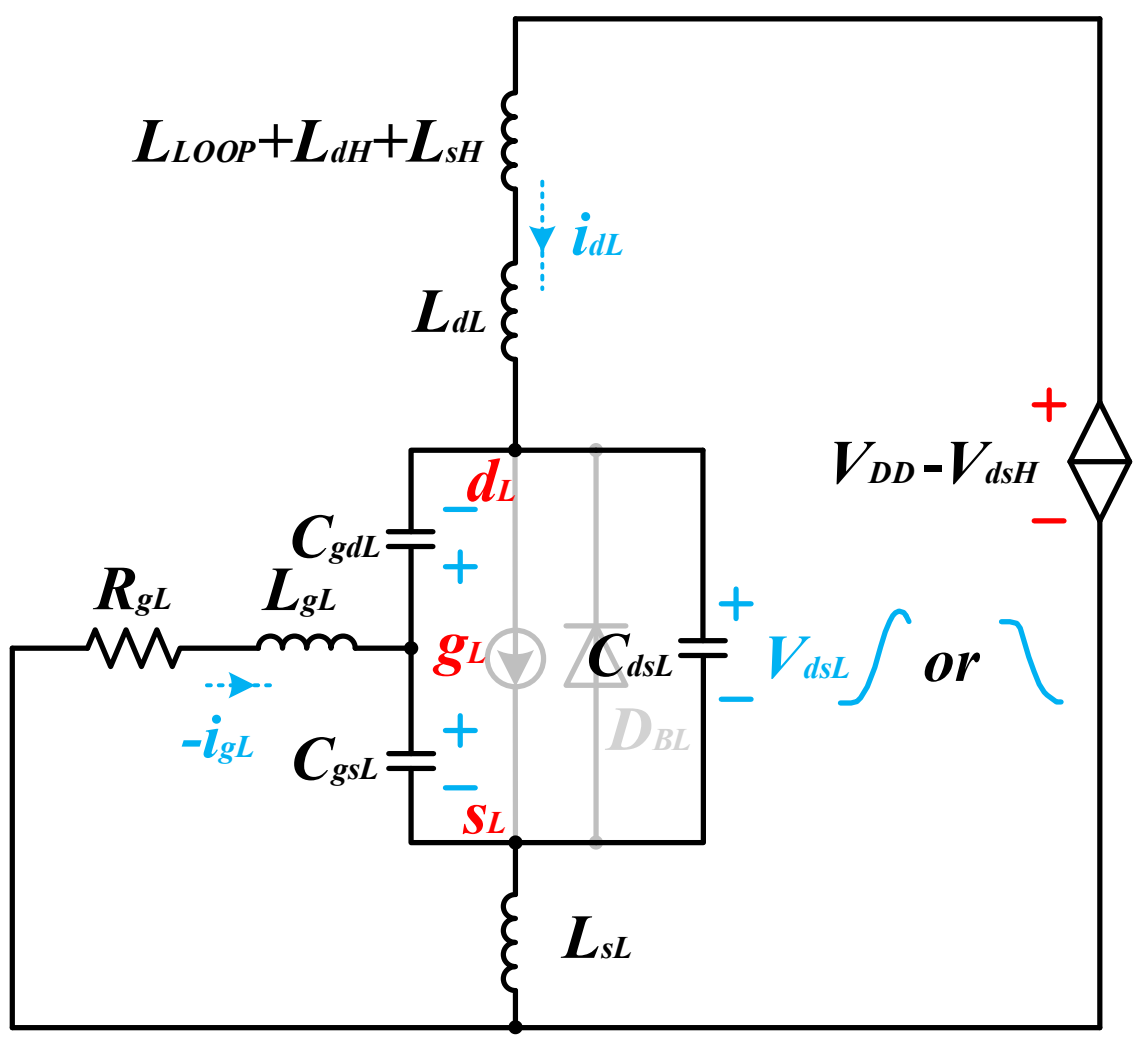

Figure 11. Equivalent circuits for $V_{d s L}$ analysis circuit.

Furthermore, for the turn-on voltage switching stage (Stage 3), the rising of $V_{d s L}$ is accompanied by the reverse recovery process of $D_{B L}$ (the blocking voltage building up), there are the initial value and rate of the drain current, $\left.i_{d L}\right|_{t=t 2}$ and $d i_{d L} /\left.d t\right|_{t=t 2}$, at the 
end time of Stage 2, which can be obtained from Section 2. For the whole structs of $Q_{L}$, the reverse recovery model of the independently packaged freewheeling diode applied in Refs. $[21,38]$ is inadequate to describe the drain-source voltage building process. It is because the freewheeling diode is inversely paralleled with the SiC MOSFET; hence, all the parasitic capacitances need to be considered. The independent barrier capacitance charge model will bring about dynamic accumulation errors for the response of $V_{d s L}$. To sum up, combining with the bridge-arm structure, all the factors of the reverse recovery current and the parasitic elements should be considered as a whole in this stage.

The capacitances, $C_{g s L}, C_{g d L}$, and $C_{d s L}$ are in delta connection and the driver loop is paralleled with $C_{g s L}$ and $L_{S L}$. The delta connection of capacitances can be transformed into the star connection, $C_{g L}, C_{d L}$, and $C_{s L}$ [39], as shown in Figure 12a. The expressions of capacitances, $C_{g L}, C_{d L}$, and $C_{s L}$ are given as follows

$$
\left\{\begin{array}{l}
C_{g L}=C_{g s L}+C_{g d L}+C_{g s L} C_{g d L} / C_{d s L} \\
C_{s L}=C_{g s L}+C_{d s L}+C_{g s L} C_{d s L} / C_{g d L} \\
C_{d L}=C_{g d L}+C_{d s L}+C_{g d L} C_{d s L} / C_{g s L}
\end{array}\right.
$$

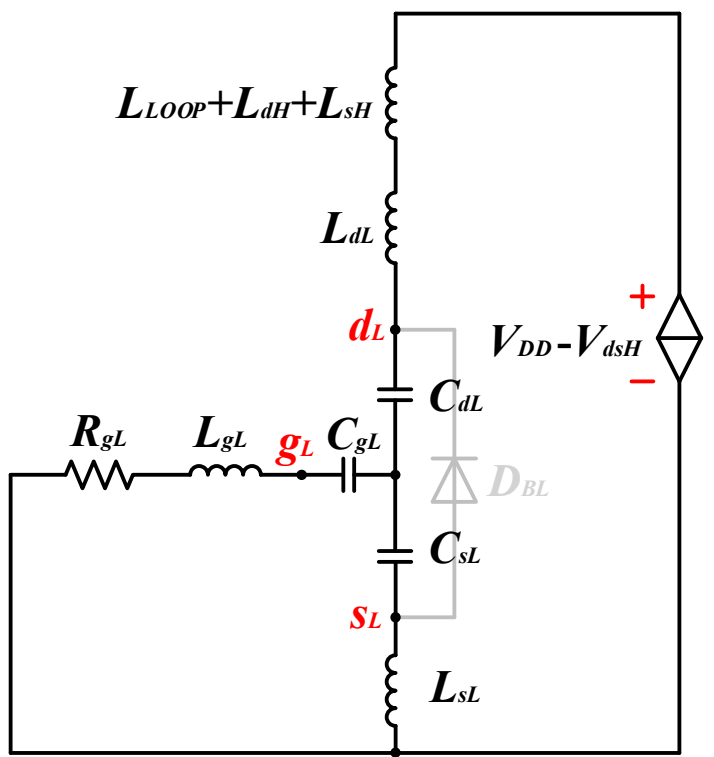

(a)

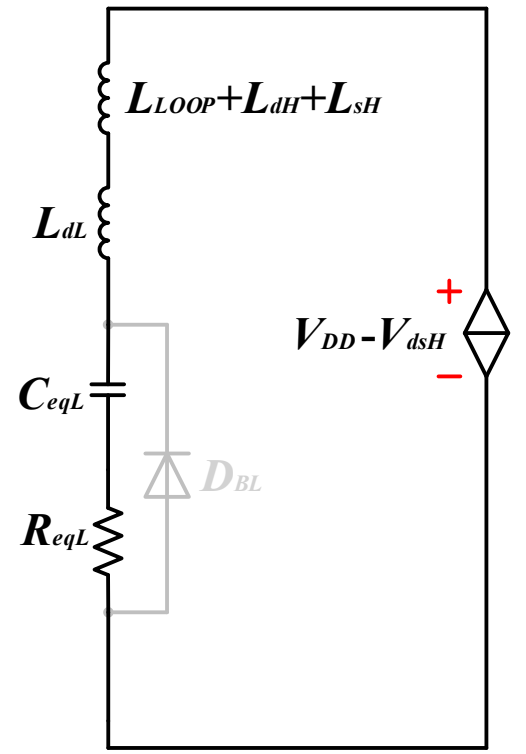

(b)

Figure 12. Simplified equivalent circuit for bridge-arm in Stage 3. (a) Star connection equivalent circuit; (b) Second-order equivalent circuit.

Considered from the perspective of the power loop, the paralleled driver loop and the $C_{s L}-L_{s L}$ branch can be further regarded as the equivalent impedance to evaluate its impact. The impedance of the driver loop can be expressed as

$$
X_{g L}=R_{g L}+j \omega_{n_{-} P} L_{g L}+\frac{1}{j \omega_{n_{-} P} C_{g L}}
$$

The impedance of the $C_{s L}-L_{S L}$ branch is expressed as

$$
X_{s L}=j \omega_{n-P} L_{s L}+\frac{1}{j \omega_{n \_} C_{s L}}
$$

where $\omega_{n_{-} P}$ is the resonance frequency of the power loop and it can be approximated by

$$
\omega_{n_{-} P} \approx \frac{1}{\sqrt{\left(L_{d H}+L_{s H}+L_{d L}+L_{s L}\right) C_{e q L}}}
$$




$$
C_{e q L}=\frac{C_{d L} C_{s L}}{C_{d L}+C_{s L}}
$$

The total equivalent impedance of the lower SiC MOSFET the power loop is

$$
X_{L}=\frac{X_{g L} \cdot X_{s L}}{X_{g L}+X_{s L}}
$$

The equivalent damping resistance is determined by the ratio of current flowing through $R_{g L}$ and the total current of the power loop, which can be expressed by Equation (30).

$$
\begin{aligned}
R_{g L_{-} e q} & =R_{g L}\left(\frac{\left|X_{L}\right|}{\left|X_{g L}\right|}\right)^{2} \\
& =R_{g L} \frac{\left(\omega_{n_{-} P} L_{S L}-\frac{1}{\omega_{n_{-} P C_{S L} L}}\right)^{2}}{R_{g L}^{2}+\left(\omega_{n_{-} P} L_{g L}-\frac{1}{\omega_{n_{-} P C_{g L}}}+\omega_{n_{-} P} L_{s L}-\frac{1}{\omega_{n_{-} P} C_{S L}}\right)^{2}}
\end{aligned}
$$

Thus, the power loop is equivalent to a second-order circuit, as shown in Figure 12b. The total equivalent resistance can be express by

$$
R_{\text {eqL }}=R_{g L \_ \text {eq }}+R_{\text {dsH_on }}+R_{L O O P}
$$

The power loop equations can be modeled as

$$
\begin{gathered}
V_{d s L}+R_{e q L} i_{d L}+\left(L_{d H}+L_{s H}+L_{d L}+L_{L O O P}\right) \frac{d i_{d L}}{d t}=V_{D D}-V_{d s H} \\
i_{d L}=C_{e q L} \frac{d V_{d s L}}{d t}
\end{gathered}
$$

It is worth noting that the nonlinear changes of $C_{g}, C_{d}$, and $C_{s}$ also need to consider. They can be derived according to the curves of interelectrode capacitances, which is shown in Figure 13.

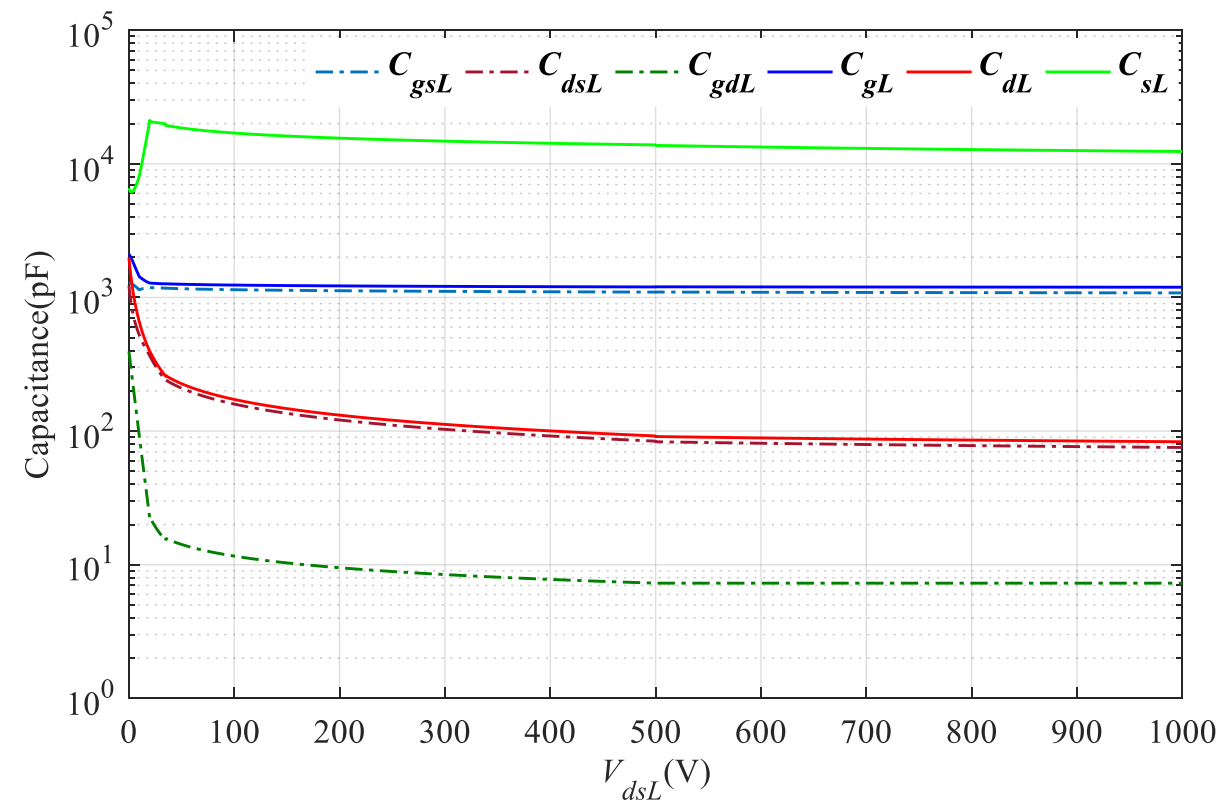

Figure 13. Comparison between the equivalent capacitances and the interelectrode capacitances.

When $V_{d s H}$ drops to the on-state voltage $V_{d s H_{-} o n}$ (Stage 4), although $Q_{H}$ enters the ohmic region, $V_{d s L}$ of $Q_{L}$ will continue to oscillate after it rises to $V_{D D}$ due to the RLC characteristic of the power loop. The ideal voltage division given in [24] cannot be used 
here, since it does not take into account the power loop oscillation. In Stage 4, the supply voltage is $V_{D D^{-}} V_{d s H_{-} o n}$ and the equivalent circuit is shown in Figure 14. The structure of the equivalent circuit is the same as in the previous stage and the expressions of $C_{e q L}$ and $R_{e q L}$ can also be described as (28) and (30). The power loop equation is transformed from (32) to (34).

$$
V_{d s L}+R_{e q} i_{d L}+\left(L_{d H}+L_{s H}+L_{d L}+L_{L O O P}\right) \frac{d i_{d L}}{d t}=V_{D D}-V_{d s H_{-} o n}
$$

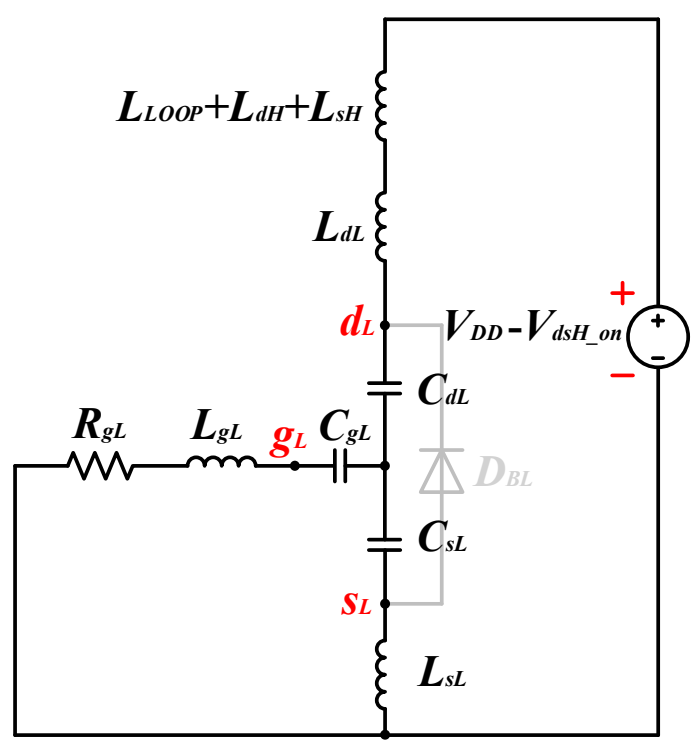

(a)

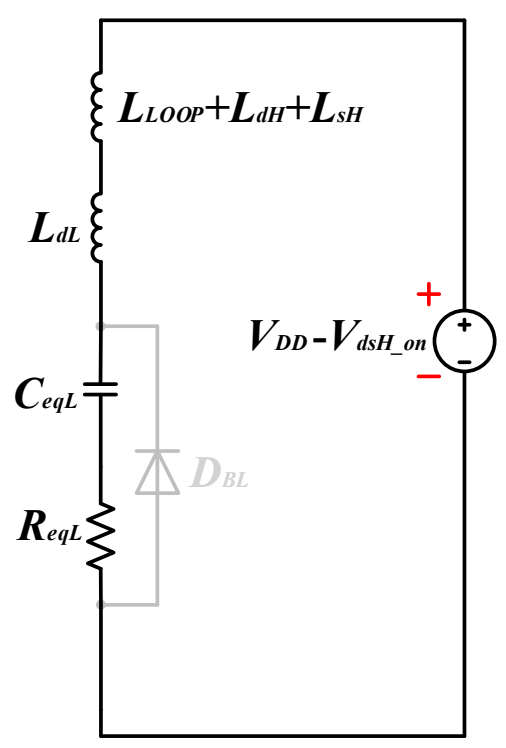

(b)

Figure 14. Simplified equivalent circuit for bridge-arm in Stage 4. (a) Star connection equivalent circuit; (b) Second-order equivalent circuit.

In the turn-off process, the circuit topologies transformations for the analysis of the voltage and current switching stages are the same as those in the turn-on stages. The voltage and current switching rates are still controlled by $Q_{H}$. The only difference is that the initial value and rate of the drain current are zero at the beginning of Stage 6.

\subsection{Analytical Model of Crosstalk}

During the current switching stages (Stage 2 and Stage 7), $V_{d s L}$ does not change, only the induced voltage on the common source parasitic inductance, $L_{s L}$, affects the driver loop, combining the equations of the driver loop (2), (3) with the equations of the channel currents (11)-(14), the response of $V_{g s L}$ is correlated with $V_{g s H}$ ultimately:

$$
\begin{gathered}
L_{g L} C_{g s L} \frac{d^{2} V_{g s L}}{d t^{2}}+R_{g L} C_{g s L} \frac{d V_{g s L}}{d t}+V_{g s L}=-L_{s L} g_{f} \frac{d V_{g s H}}{d t} \\
\left(L_{g H}+L_{s H}\right) C_{g s H} \frac{d^{2} V_{g s H}}{d t^{2}}+\left(R_{g H} C_{g s H}+L_{s H} g_{f}\right) \frac{d V_{g s H}}{d t}+V_{g s H}=V_{G A T E}
\end{gathered}
$$

During the voltage switching stages (Stage 3 and Stage 6), it can be generalized that the initial state of the drain current, the nonlinear switching rate of the drain-source voltage, the parasitic inductances of the power loop and common source, and the nonlinear characteristics of the interelectrode capacitances affect the response of $V_{d s L}$. Through the analysis in section A. All these factors can be further directly or indirectly linked with the response of $i_{d L}$. $i_{d L}$ becomes a key vinculum to reflect the impacts of the voltage switching. The correlations are summarized in Figure 15. 


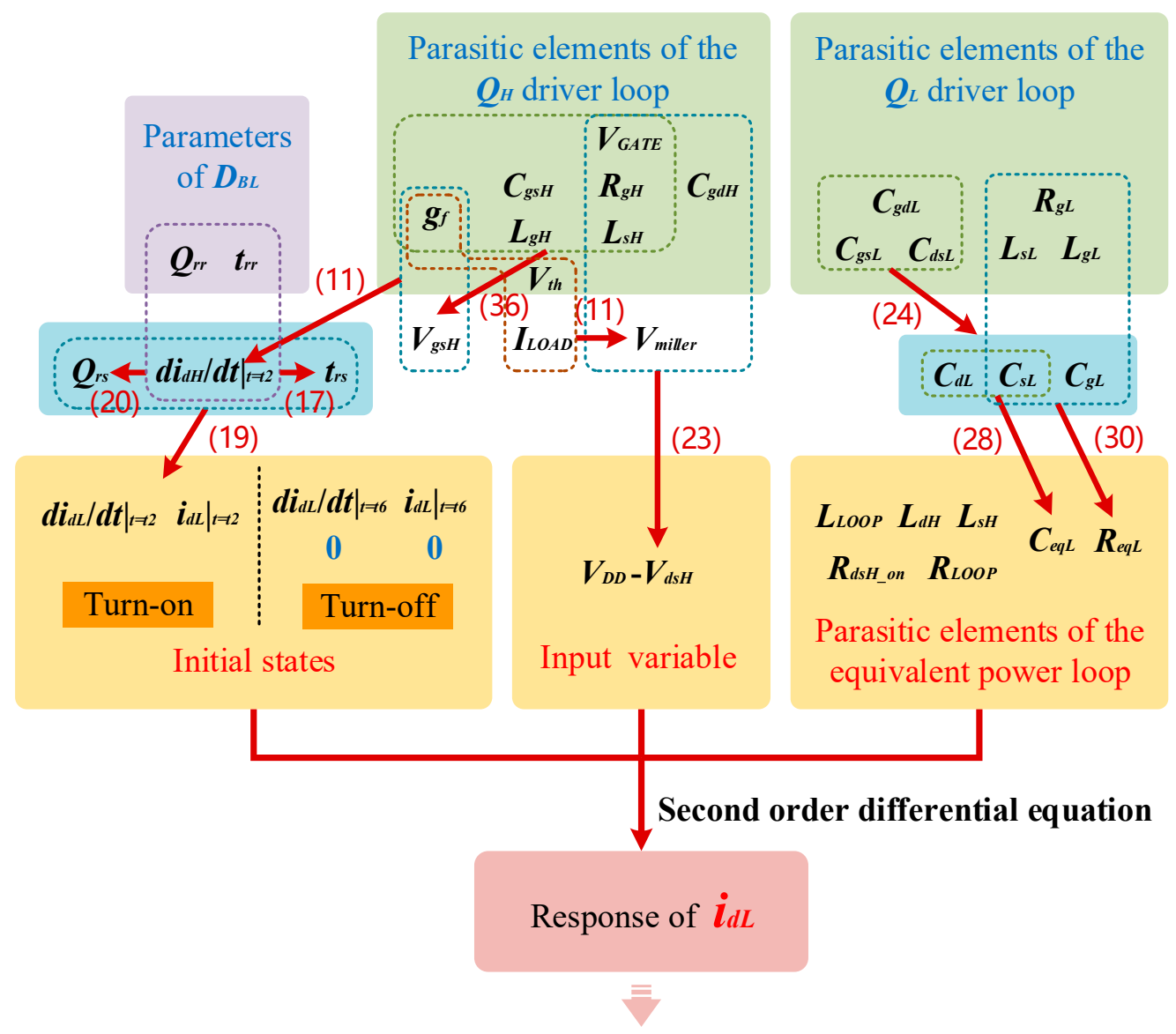

Figure 15. Relationships of $i_{d L}$ with the parameters of the bridge-arm.

Combining (31)-(33) with (23), $i_{d L}$ can be expressed as

$C_{e q L}\left(L_{d H}+L_{s H}+L_{d L}+L_{L O O P}\right) \frac{d^{2} i_{d L}}{d t^{2}}+C_{e q L}\left(R_{e q L}+\frac{L_{s H}}{R_{g H} C_{g d H}}\right) \frac{d i_{d L}}{d t}+i_{d L}=\frac{\left(V_{G A T E}-V_{\text {miller }}\right)}{R_{g H} C_{g d H}} C_{e q L}$

After $V_{d s L}$ rises to $V_{D D}$ in the turn-on process, $i_{d L}$ is expressed as

$$
C_{e q L}\left(L_{d H}+L_{s H}+L_{d L}+L_{L O O P}\right) \frac{d^{2} i_{d L}}{d t^{2}}+C_{e q L} R_{e q L} \frac{d i_{d L}}{d t}+i_{d L}=0
$$

Next, $i_{d L}$ is used as an input source to analyze its influence on the crosstalk of the driver loop. The interelectrode capacitances can be converted into two branches with the delta-star transformation, which helps simplify the analysis. The current flowing through $C_{d L}$ is $i_{d L}$. From the perspective of the driver loop, the crosstalk response is converted to the voltage difference between $C_{s L}$ and $C_{g L}$ on the two branches. The equivalent circuit is shown in Figure 16 and the equations can be established as

$$
\begin{aligned}
V_{g s L} & =V_{g L}+V_{s L} \\
i_{d L} & =i_{s L}-i_{g L} \\
i_{g L} & =C_{g L} \frac{d V_{g L}}{d t} \\
i_{s L} & =C_{s L} \frac{d V_{s L}}{d t} \\
-V_{g L}-R_{g L} i_{g L}- & L_{g L} \frac{d i_{g L}}{d t}=V_{s L}+L_{s L} \frac{d i_{s L}}{d t}
\end{aligned}
$$




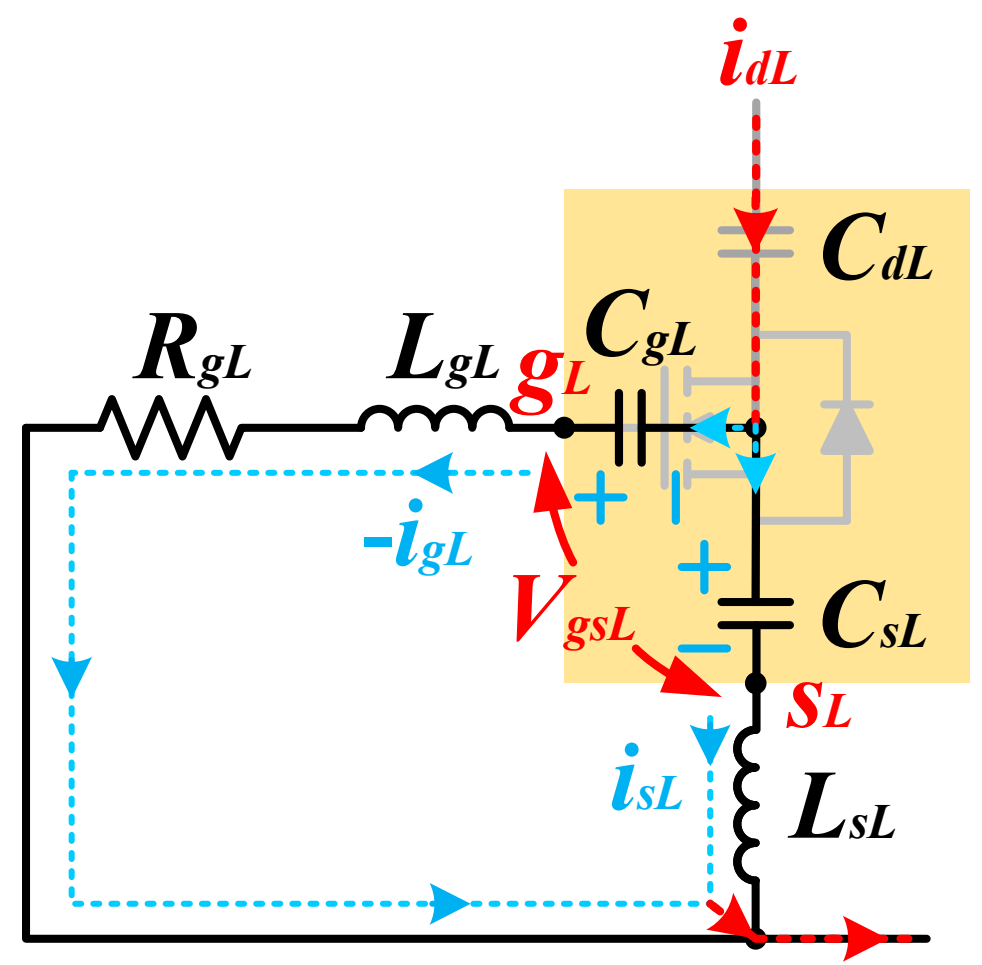

Figure 16. Equivalent circuit for the driver loop of $Q_{L}$.

Therefore, the relationship of $i_{g L}$ and $i_{d L}$ can be obtained as follow

$$
\left(L_{g L}+L_{s L}\right) C_{e q L_{-} d} \frac{d^{2} i_{g L}}{d t^{2}}+R_{g L} C_{e q L_{-} d} \frac{d i_{g L}}{d t}+i_{g L}=-L_{s L} C_{e q L \_} \frac{d^{2} i_{d L}}{d t^{2}}+\frac{C_{e q L \_} d}{C_{s L}} i_{d L}
$$

$V_{g s L}$ can be expressed by

$$
V_{g S L}=-R_{g L} i_{g L}-\left(L_{g L}+L_{s L}\right) \frac{d i_{g L}}{d t}-L_{s L} \frac{d i_{d L}}{d t}
$$

where $C_{e q L} d=C_{g L} C_{s L} /\left(C_{g L}+C_{s L}\right)$. Finally, the response of $V_{g s L}$ can be deducted by correlating the differential Equations (37), (44), and (45).

So far, the crosstalk responses of the turn-on and turn-off processes are fully expressed in the time domain. The Equations (35), (44), and (45) can be discretized and brought into MATLAB, where the directly correlated variable responses are expressed by Equations (36)-(38). Based on the software, the whole simulation waveforms of $V_{g s L}$ can be presented minutely and accurately. The detailed simulation and experimental verification are described in the next section.

\section{Simulation and Experimental Verification}

To verify the analysis and modeling method in the previous section, a typical double pulse test circuit platform is built, Figure 17 shows the photograph of the test platform. Two 1200 V/36 A SiC MOSFETs C2M0080120D, from Wolfspeed corporation, are connected in series as the upper and lower switching devices of the bridge-arm, a $12 \mu \mathrm{H}$ ferrite-core inductance is connected in parallel with the lower SiC MOSFET as the load inductance. The test circuit is supported by $600 \mathrm{~V}$ DC supply voltage, multiple groups of $10 \mu \mathrm{F}$ capacitances are connected in parallel with the DC input terminal to ensure the voltage stability. The initial parameters of the circuit model are listed in Table 2. Some fixed parameter values were obtained through the datasheet, including $Q_{r r}, g_{f}, R_{g_{-} i n}$, and $R_{d s_{-} o n}$. The values of parasitic inductances were implemented by an ANSYS Q3D Extractor finite-element analysis (FEA) simulation, which includes internal pins of the devices and the printed 
circuit board (PCB) layout [40], The external pins of the devices and the connecting leads of the power loop were measured by an Agilent 4395A impedance analyzer.

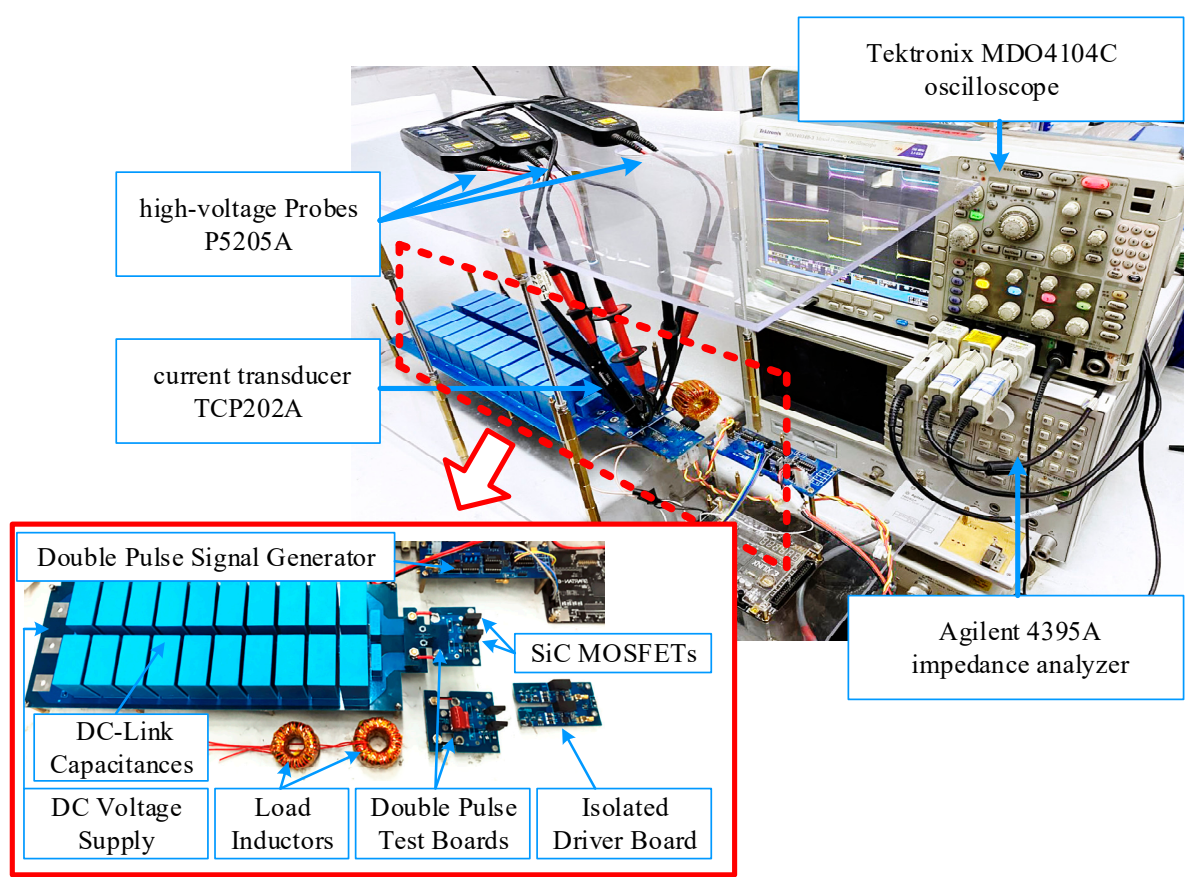

Figure 17. Photograph of the double pulse test platform.

Table 2. Fixed Parameters Setup.

\begin{tabular}{|c|c|c|c|c|}
\hline Component & Parameter & Value & Parameter & Value \\
\hline \multirow{2}{*}{ Power circuit setting } & $V_{D D}$ & $600 \mathrm{~V}$ & $R_{L O O P}$ & $230 \mathrm{~m} \Omega$ \\
\hline & $I_{D D}$ & $20 \mathrm{~A}$ & $L_{L O O P}$ & $32 \mathrm{nH}$ \\
\hline \multirow{12}{*}{$\begin{array}{l}\text { Fixed parameters of upper } \\
\text { and lower SiC MOSFET } \\
\text { (C2M0080120D) }\end{array}$} & $V_{t h}$ & $3.3 \mathrm{~V}$ & $Q_{r r}$ & $192 \mathrm{nC}$ \\
\hline & $V_{G A T E}$ & $+20 \mathrm{~V} /-5 \mathrm{~V}$ & $V_{\text {GATE }}$ & $-5 \mathrm{~V}$ \\
\hline & $g_{f}$ & $8.1 \mathrm{~S}$ & $V_{S D}$ & $3.3 \mathrm{~V}$ \\
\hline & $R_{d s H_{\text {on }} n}$ & $40 \mathrm{~m} \Omega$ & $R_{d s H \_o n}$ & $40 \mathrm{~m} \Omega$ \\
\hline & $R_{g H \_e x}$ & $10 \Omega$ & $R_{g L \_} e x$ & $10 \Omega$ \\
\hline & $R_{g H \_i n}$ & $4.6 \Omega$ & $R_{g L \_i n}$ & $4.6 \Omega$ \\
\hline & $L_{g H \_i n}$ & $1.1 \mathrm{nH}$ & $L_{g L \_ \text {in }}$ & $1.7 \mathrm{nH}$ \\
\hline & $L_{g H \_}$ex & $3.0 \mathrm{nH}$ & $L_{g L \_e x}$ & $2.8 \mathrm{nH}$ \\
\hline & $L_{S H}{ }_{-i n}$ & $1.3 \mathrm{nH}$ & $L_{S L \_i n}$ & $1.3 \mathrm{nH}$ \\
\hline & $L_{S H \_}$ex & $3.7 \mathrm{nH}$ & $L_{S L \_e x}$ & $3.4 \mathrm{nH}$ \\
\hline & $L_{d H \_i n}$ & $1.2 \mathrm{nH}$ & $L_{d L \_i n}$ & $1.1 \mathrm{nH}$ \\
\hline & $L_{d H \_e x}$ & $3.3 \mathrm{nH}$ & $L_{d L \_e x}$ & $3.6 \mathrm{nH}$ \\
\hline
\end{tabular}

The circuit setting and the parameter values in the simulation calculations are based on this real circuit experiment platform within a reasonable range. The nonlinear capacitances are piecewise fitted according to the datasheet, the piecewise parameters are listed in Table 3. This piecewise fitting model can be invoked as independent computing units based on the values of $V_{d s H}$ and $V_{d s L}$ to obtain the precise value in every discrete computing period. 
Table 3. Parameters of Capacitance Piecewise Fitting Model.

\begin{tabular}{|c|c|c|c|c|c|c|c|}
\hline \multirow[b]{2}{*}{ Capacitance } & \multicolumn{2}{|c|}{ Parameter } & \multicolumn{5}{|c|}{$V_{d s}$ Range (V) } \\
\hline & $C_{j 0}(\mathrm{pF})$ & Fitting Parameter & $0-9$ & $9-20$ & $20-35$ & $35-500$ & $500-1200$ \\
\hline \multirow{2}{*}{$C_{i s s}$} & \multirow{2}{*}{1700} & $V_{j}$ & 20 & \multirow{2}{*}{\multicolumn{3}{|c|}{$\begin{array}{c}1 \times 10^{-4} \\
0.028\end{array}$}} & $1 \times 10^{-6}$ \\
\hline & & $m$ & 0.8 & & & & 0.0125 \\
\hline \multirow{2}{*}{$C_{\text {oss }}$} & \multirow{2}{*}{1650} & $V_{j}$ & \multirow{2}{*}{\multicolumn{3}{|c|}{$\begin{array}{c}5 \\
0.9\end{array}$}} & 0.3 & $1 \times 10^{-7}$ \\
\hline & & $m$ & & & & 0.39 & 0.13 \\
\hline \multirow{2}{*}{$C_{r S S}$} & \multirow{2}{*}{400} & $V_{j}$ & 350 & 300 & 0.4 & $5 \times 10^{-4}$ & $3 \times 10^{-4}$ \\
\hline & & $m$ & 45 & 43 & 0.73 & 0.29 & 0.29 \\
\hline
\end{tabular}

In order to distinguish the response stages and accurately divide the related factors of crosstalk in different stages, the terminal value of the current response in Stage 2 and Stage 7 and the voltage response in Stage 3 and Stage 6 need to be identified as the switching judgment conditions. They are listed in Table 4 and the equations also need to be added to the simulation calculation program.

Table 4. Switching Time and Calculations of Judgement Conditions.

\begin{tabular}{|c|c|c|c|c|c|c|}
\hline $\begin{array}{l}\text { Switching } \\
\text { Time }\end{array}$ & $t 1$ & $t 2$ & $t 3$ & $t 6$ & $t 7$ & t8 \\
\hline $\begin{array}{l}\text { Judgement } \\
\text { condition }\end{array}$ & $V_{g s H}>V_{t h}$ & $i_{d L}>I_{r r_{-} \text {peak }}$ & $V_{d s H}<V_{t h}$ & $V_{g s H}<V_{\text {miller }}$ & $V_{d s L}<0$ & $i_{d L}<-i_{L O A D}$ \\
\hline $\begin{array}{l}\text { Calculate } \\
\text { equations }\end{array}$ & $\begin{array}{c}(11),(12), \text { and } \\
(13) \\
V_{i d s H}=0\end{array}$ & $\begin{array}{l}(10),(11),(12), \\
\text { and (13) }\end{array}$ & $\begin{array}{c}(22) \\
\text { integrated } \\
\text { computation } \\
\text { from } t 2\end{array}$ & $\begin{array}{c}(11),(12), \text { and } \\
(13) \\
V_{i d s H}=0\end{array}$ & $\begin{array}{c}(22) \\
\text { integrated } \\
\text { computation } \\
\text { from } t 6\end{array}$ & $\begin{array}{l}(10),(11),(12), \\
\text { and (13) }\end{array}$ \\
\hline Initial states & - & $\begin{array}{c}\left.V_{g s H}\right|_{t=t 1} \\
d V_{g s H} /\left.d t\right|_{t=t 1}\end{array}$ & $\begin{array}{c}V_{D D} \\
\left.i_{d L}\right|_{t=t 2} \\
d_{i d L} /\left.d t\right|_{t=t 2}\end{array}$ & - & - & $\begin{array}{c}\left.i_{d L}\right|_{t=t 7} \\
d_{i d L} /\left.d t\right|_{t=t 7}\end{array}$ \\
\hline
\end{tabular}

According to the piecewise conditions, the crosstalk response can be calculated stageby-stage with iterative method, by discretizing the equations, it is convenient to obtain the numerical solution of the variable responses. The simulated waveforms of crosstalk, when $V_{D D}=600 \mathrm{~V}$ and $I_{L O A D}=20 \mathrm{~A}$, are shown in Figure 18. In addition, the calculation results according to the model presented in the literature [17,24], and the theoretical extremum of crosstalk [15] are also placed on the same coordinates to horizontal compare the differences.

It can be seen from Figure 18, during the current switching stages in the turn-on process, there is a drop on $V_{g s L}$, which reflects the impacts of the common source parasitic inductance with the rapid current changes. However, the calculations in Refs. [17,24] do not consider the influence of the parasitic inductances, so that there is a significant difference in the negative fluctuations of the crosstalk. There are trends in our data to suggest that the positive peak value of crosstalk is higher compared with the calculation in Ref. [24]. $V_{g s L}$ will continue to rise and start oscillating after the voltage switching process, which embodies the coupling effect of the oscillation caused by the parasitic inductance and parasitic capacitance of the power loop on the crosstalk. In the turn-off process, the negative peak value of the crosstalk proposed in this paper is higher than the calculated value in Ref. [24], which is also due to the influence of parasitic inductance. More specifically, during the voltage switching stages (Stage 6), the parasitic capacitances of $Q_{L}$ are discharged with the decrease of $V_{d s L}$, the displacement current of $C_{g d L}$ induces a negative fluctuation in $V_{g s L}$, but the discharge current for $C_{d s L}$ starts to increase from zero, which causes a negative induced voltage on $L_{s L}$, thus, it will suppress the negative fluctuation of $V_{g s L}$. Further, the simulation results of the crosstalk peak values given in this paper and Ref. [24] are both significantly larger than the calculation results with constant capacitances, these all 
indicate that the nonlinear parasitic capacitances have obvious influences on the crosstalk evaluation.

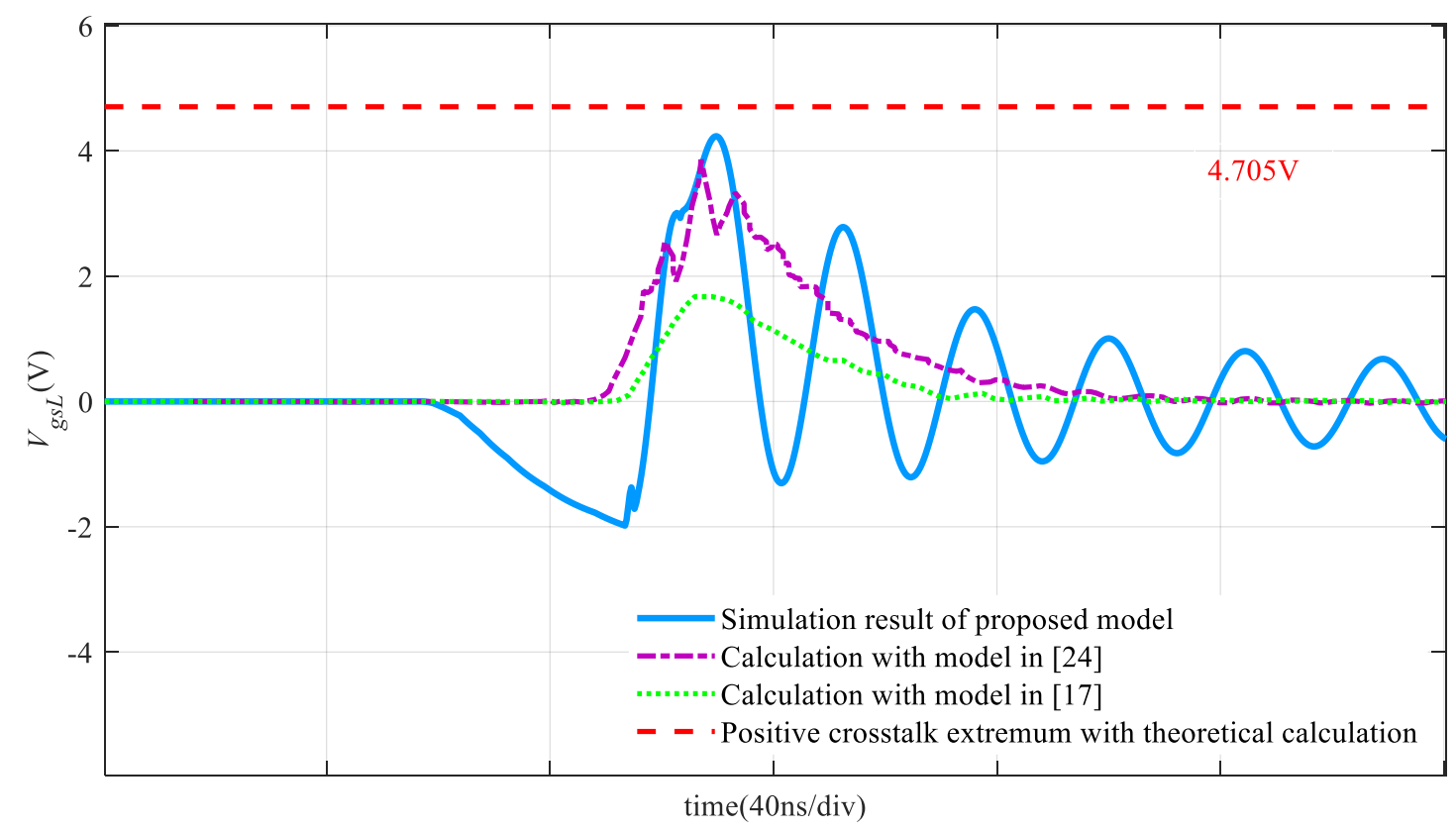

(a)

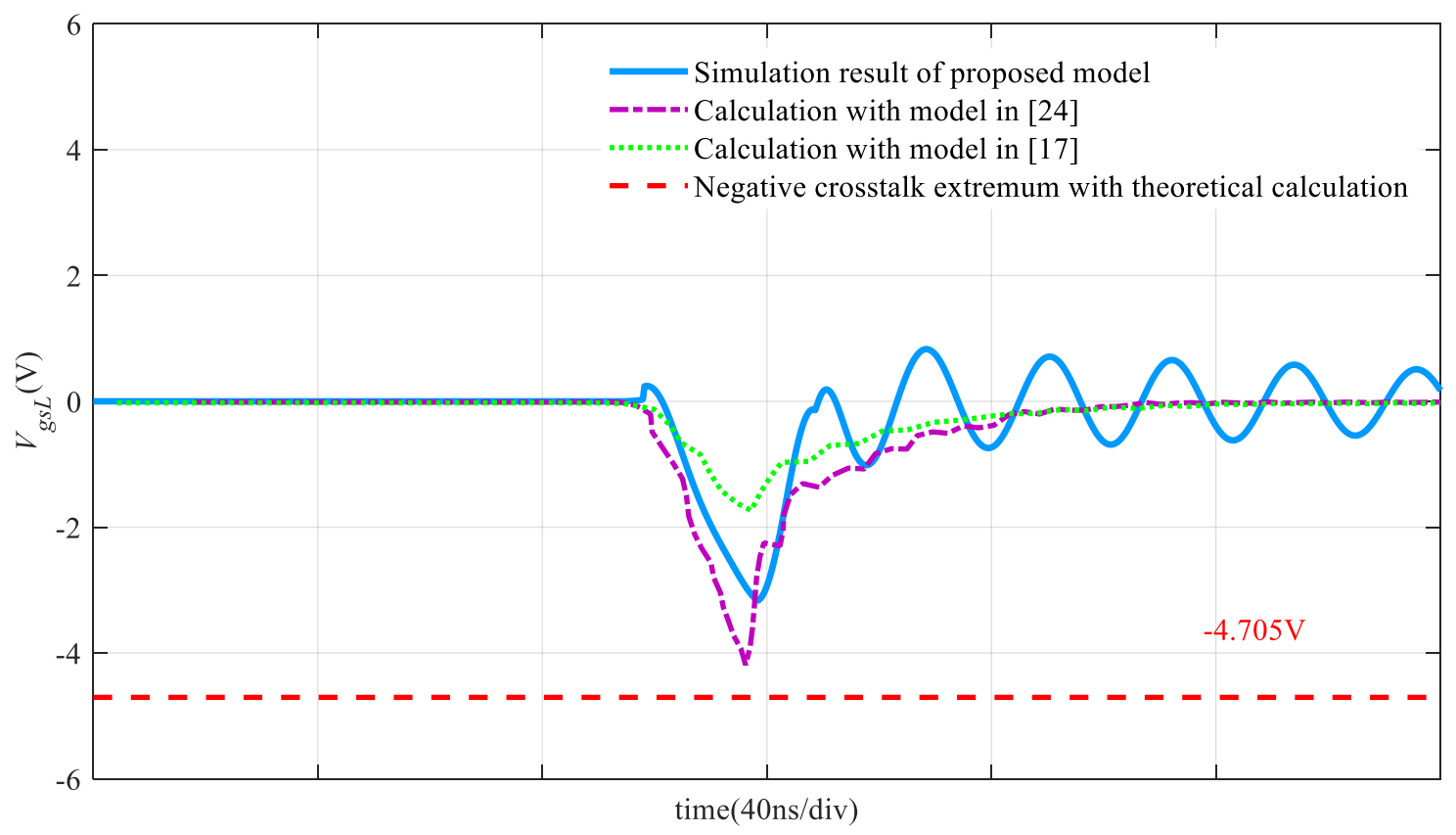

(b)

Figure 18. Crosstalk waveforms provided by the proposed analytical model, the analytical model in [17] the analytical model in [24], and the extremum calculated by theoretical calculation. (a) turn-on process. (b) turn-off process.

The experimental platform is employed to verify the correctness of the proposed model. In the experimental test, since there is no any crosstalk suppression method, to prevent spurious trigger, the negative driver voltage level of $Q_{L}$ is set at $-5 \mathrm{~V}$. Adjusting the negative voltage bias does not have a significant effect on the responses of crosstalk voltage [24]. The double pulse trigger signal position is adjusted to set the load current at $20 \mathrm{~A}$. The first turn-off and the second turn-on waveforms are extracted in this paper due to the drain current value almost maintain the same at the switching time, as is shown in 
Figure 19. Figure 20 compares the experiment results and the simulation results of crosstalk waveforms in the turn-on and turn-off process, the experimental data are uniformly added $5 \mathrm{~V}$ to facilitate the comparisons. The red lines represent the experiment waveforms and the blue lines represent the simulation waveforms.

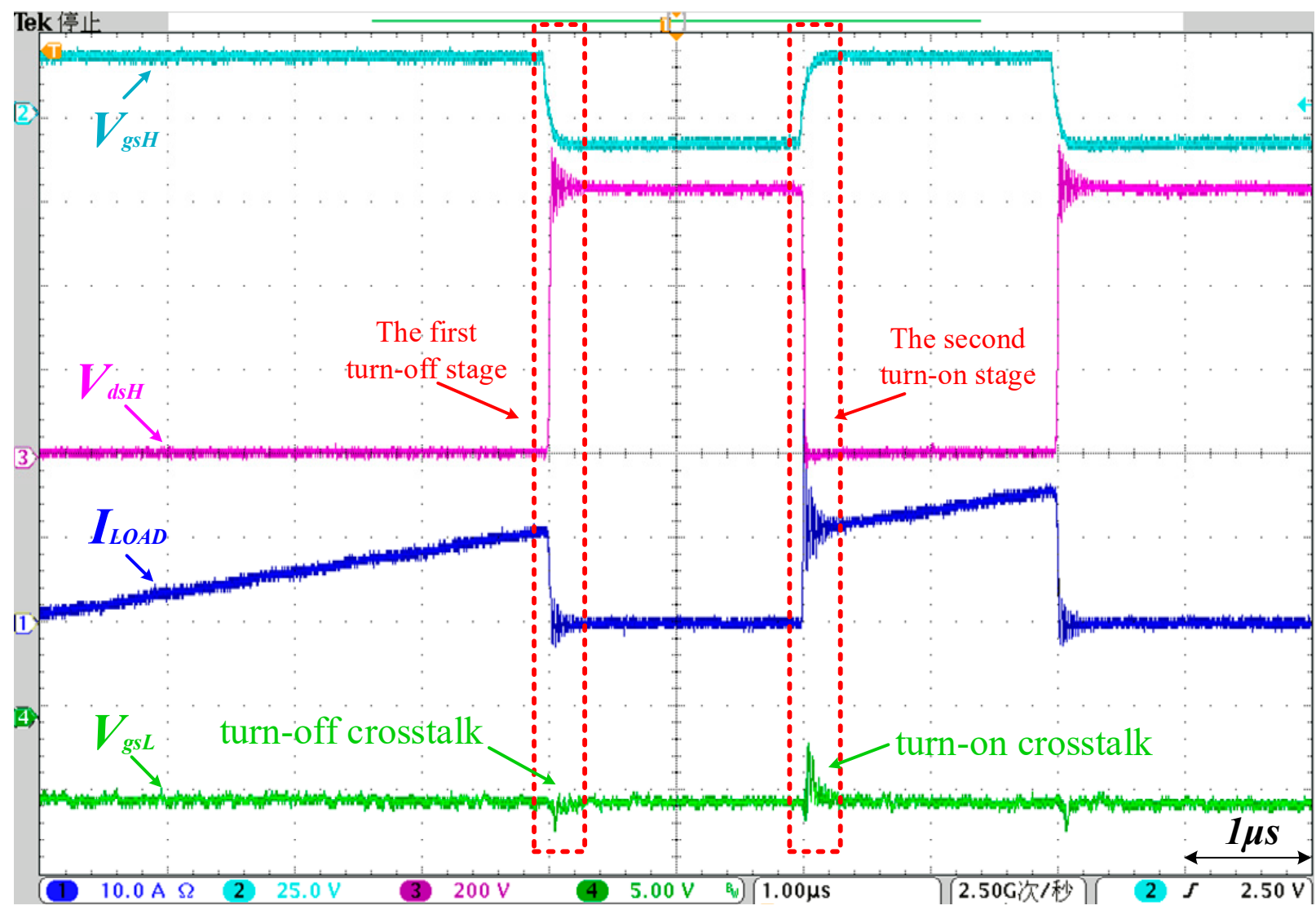

Figure 19. Double plus test waveforms.

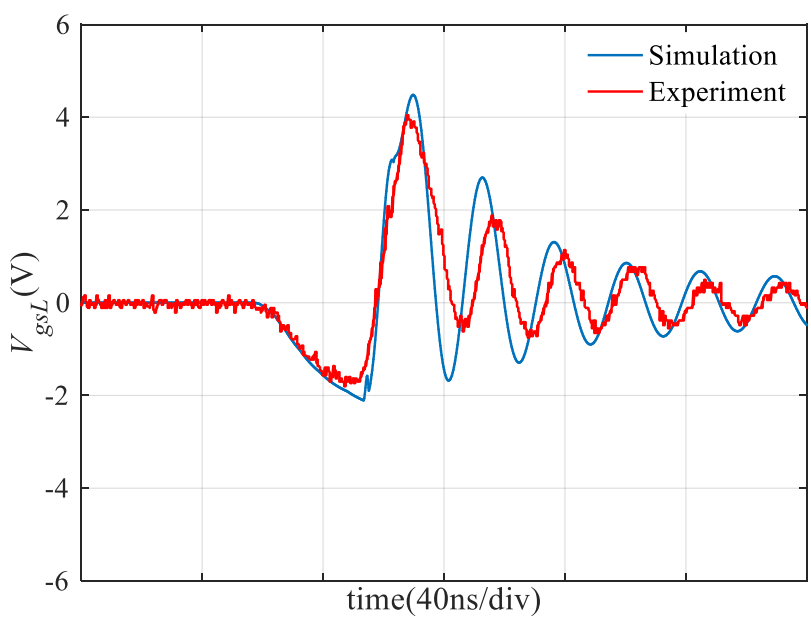

(a)

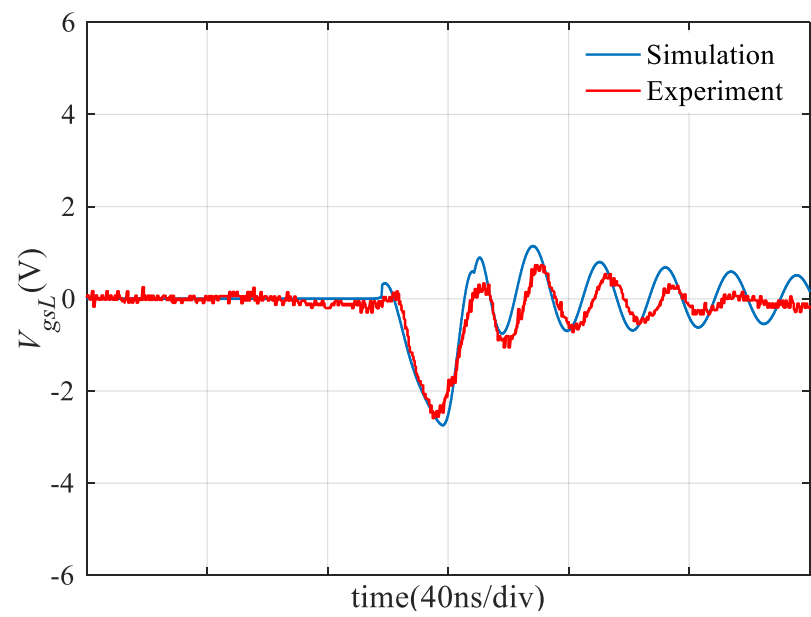

(b)

Figure 20. Comparison of simulation and experimental waveforms of crosstalk in (a) turn-on process, (b) turn-off process.

It is apparent that the proposed analytical model is more detailed and matches better with the experimental results (in terms of the voltage drop in Stage 2, the oscillations after the voltage switching, and the peak values response) than models in Refs. $[17,24]$. There 
are deviations between the simulation and actual results of the oscillation frequencies, it is because the measurement deviation of parasitic inductances and equivalent resistance of the circuit, and the model of the nonlinear capacitances is difficult to maintain a high consistency with the data in the datasheet. These deviations are inevitable but the variation trends can still be correctly inferred.

According to the previous analysis, the variables that directly impact the crosstalk are the current and voltage switching rates and they are closely related to the driver capability of the active-controlled device. In the simulations and experiments, adjusting the driver resistant $R_{g H}$ to change the switching rate of the bridge-arm, this method can adjust both voltage and current switching rates according to (11)-(14) and (23). The simulations and experiments waveforms of crosstalk are shown in Figure 21, and the trend comparisons of the relative crosstalk peak values are provided in Figure 22.

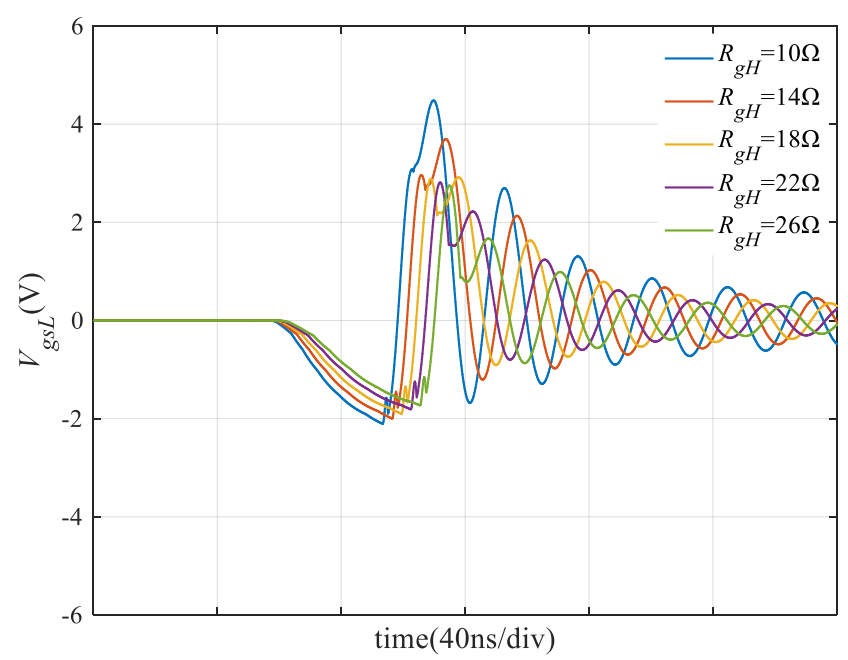

(a)

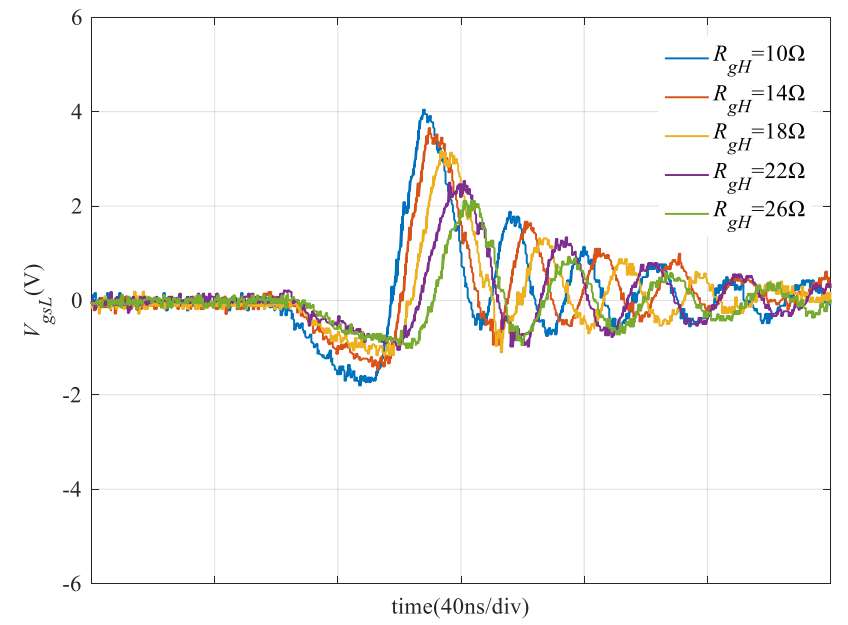

(c)

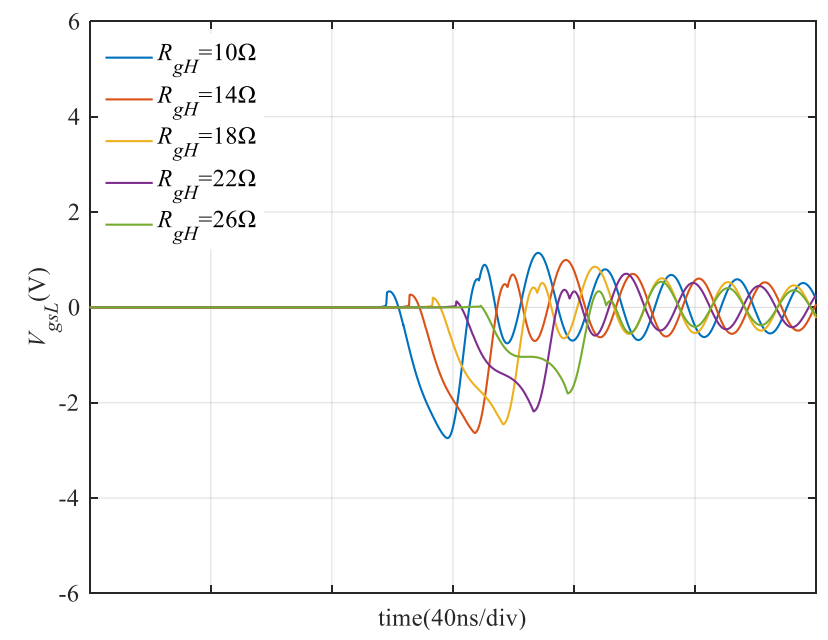

(b)

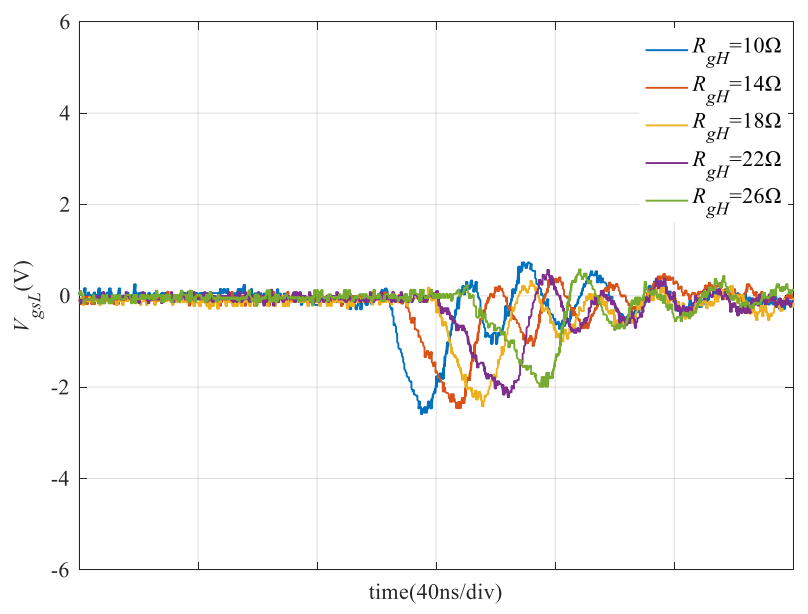

(d)

Figure 21. Comparison of simulation and experimental waveforms of crosstalk with different $R_{g H} \cdot(\mathbf{a}, \mathbf{b})$ : simulation. (c,d): experiment. Left: turn-on. Right: turn-off. 


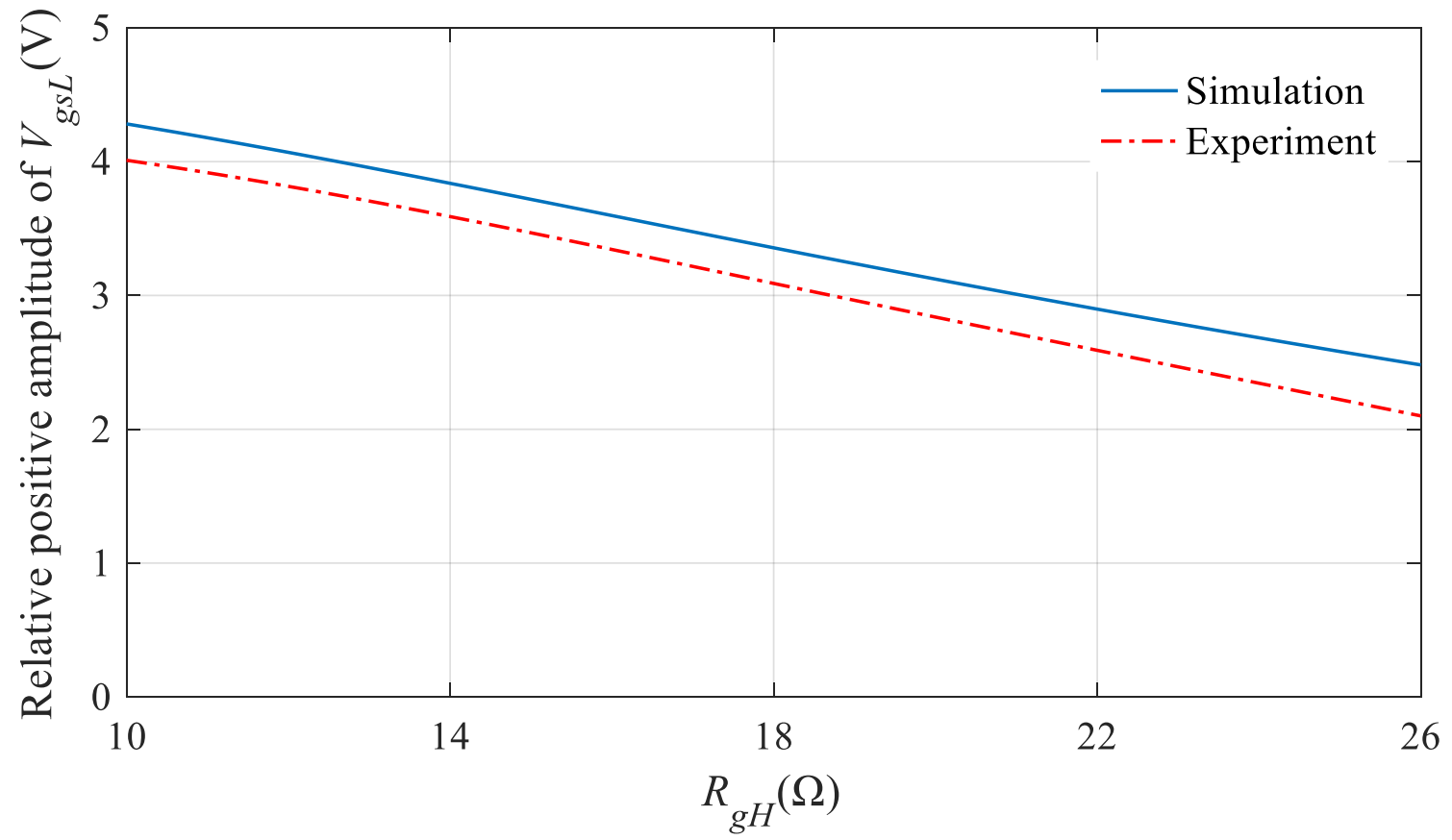

(a)

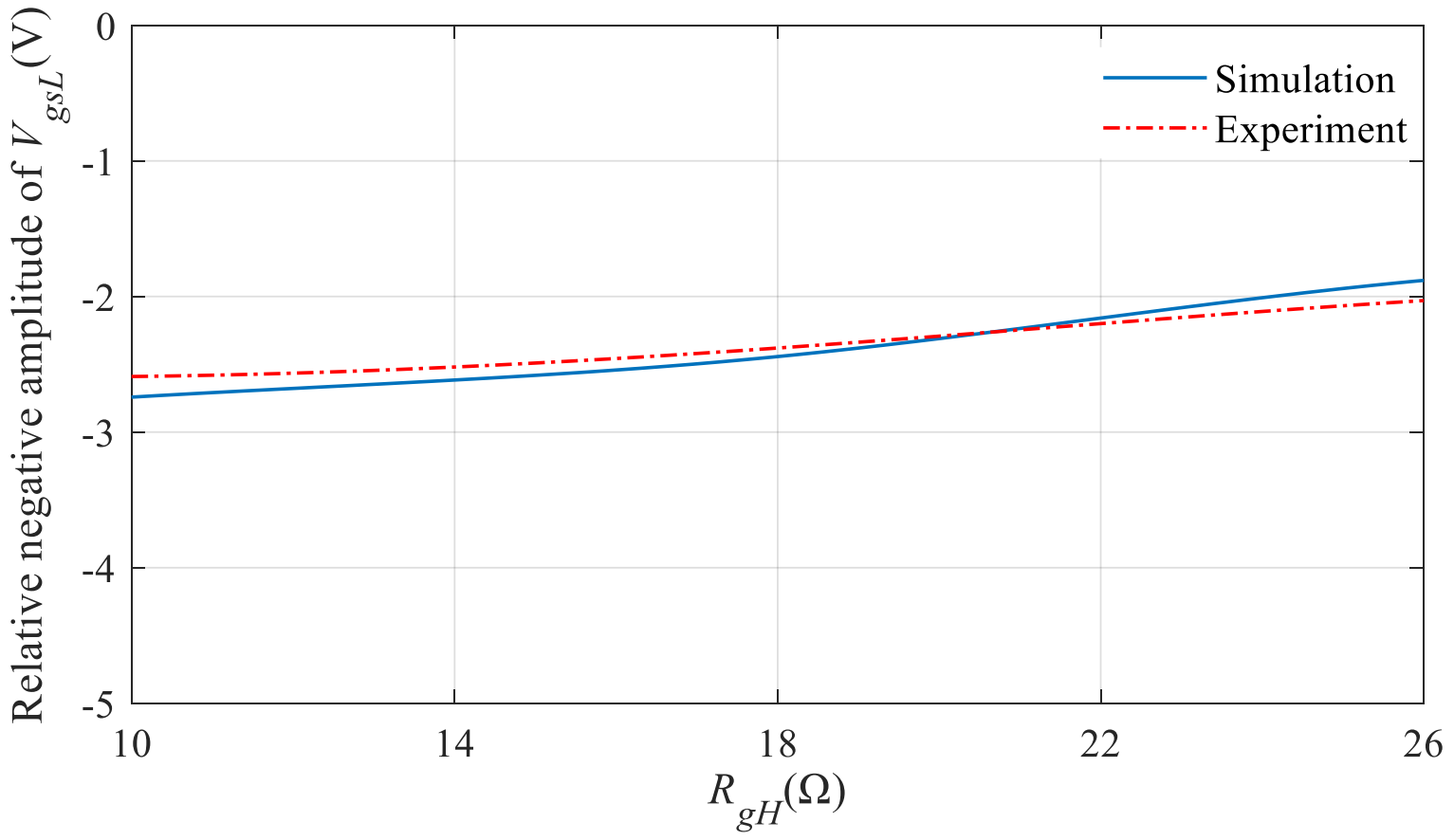

(b)

Figure 22. Comparison of simulation and experimental variation trend of relative crosstalk peak value with different $R_{g H}$. (a) turn-on. (b) turn-off.

It can be seen from Figures 21 and 22 that the experimental waveforms and the variation trends of crosstalk are in good agreement with the simulation results, which verifies the correctness and the accuracy of the proposed model with the situations where the switching rates are dynamically adjusted. However, the deviations of the peak values between the simulation and experiment results also need to be noticed. Especially in the turn-on process, the measured amplitudes of $V_{g s L}$ are smaller than the calculated values. One of the main reasons is that the voltage measurement probes can only be connected between the external terminal nodes of the gate and source, thus the internal voltages of 
parasitic elements are included in the measurement values. During the turn-on process, the voltages on the internal gate resistance, the gate parasitic inductances, and the internal source parasitic inductances are all opposite to the gate-source voltage of $C_{g s L}$, which leads to the measured peak values of $V_{g s L}$ are smaller than the calculated values. In addition, the reverse recovery current decreases rapidly from the beginning of the voltage switching stage, which produces a relatively larger negative induced voltage on the internal common source parasitic inductance. These two factors cause more obvious deviations of the crosstalk voltage in the turn-on process.

These measurement deviations are inevitable but the variation trend can still be correctly inferred, and importantly, the directly calculated analytical values of internal $V_{g s L}$ are essentially needed to evaluate the real crosstalk voltage response rather than the voltage between the external nodes. The measurement values can be considered as indirect reflections of the crosstalk. The precise values and errors of experimental measurements and simulation calculations for the relative amplitudes of the crosstalk are listed in Table 5.

Table 5. Comparison of Experiments and Simulations with Variable $R_{g H}$.

\begin{tabular}{|c|c|c|c|c|c|c|}
\hline & $\mathrm{R}_{\mathrm{gH}}(\Omega)$ & $10 \Omega$ & $14 \Omega$ & $18 \Omega$ & $22 \Omega$ & $26 \Omega$ \\
\hline \multirow{3}{*}{ Turn-on } & Relative $\mathrm{V}_{\mathrm{gsL}}$ with Simulation (V) & 4.282 & 3.838 & 3.356 & 2.898 & 2.481 \\
\hline & $\begin{array}{c}\text { Relative } \mathrm{V}_{\mathrm{gsL}} \text { with } \\
\text { Experiment }(\mathrm{V})\end{array}$ & 4.05 & 3.58 & 3.10 & 2.59 & 2.12 \\
\hline & Difference compared with experiment & $5.73 \%$ & $7.21 \%$ & $8.26 \%$ & $11.89 \%$ & $17.03 \%$ \\
\hline \multirow{3}{*}{ Turn-off } & Relative $\mathrm{V}_{\mathrm{gsL}}$ with Simulation (V) & -2.742 & -2.616 & -2.443 & -2.159 & -1.881 \\
\hline & $\begin{array}{l}\text { Relative } \mathrm{V}_{\mathrm{gsL}} \text { with } \\
\text { Experiment }(\mathrm{V})\end{array}$ & -2.59 & -2.52 & -2.38 & -2.20 & -2.02 \\
\hline & Difference compared with experiment & $5.87 \%$ & $3.81 \%$ & $2.65 \%$ & $1.86 \%$ & $6.88 \%$ \\
\hline
\end{tabular}

According to (32) and the judgment conditions of $t 3$ and $t 6$, the switching rate and duration of the voltage also changed with the different input voltage levels, which indirectly affects the crosstalk responses in the voltage switching process, especially the peak value. To verify the model in a further step, different DC voltages are applied to the double pulse test platform, while other test conditions are the same as the initial setting, the load current is kept at $20 \mathrm{~A}$ by adjusting the trigger position of the driver signal. $V_{D D}$ is increased from $200 \mathrm{~V}$ to $600 \mathrm{~V}$ with $100 \mathrm{~V}$ step. The simulation and experimental crosstalk waveforms with different $V_{D D}$ are shown in Figure 23. The precise values and errors of experimental measurements and simulation calculations for the relative amplitudes of the crosstalk are listed in Table 6.

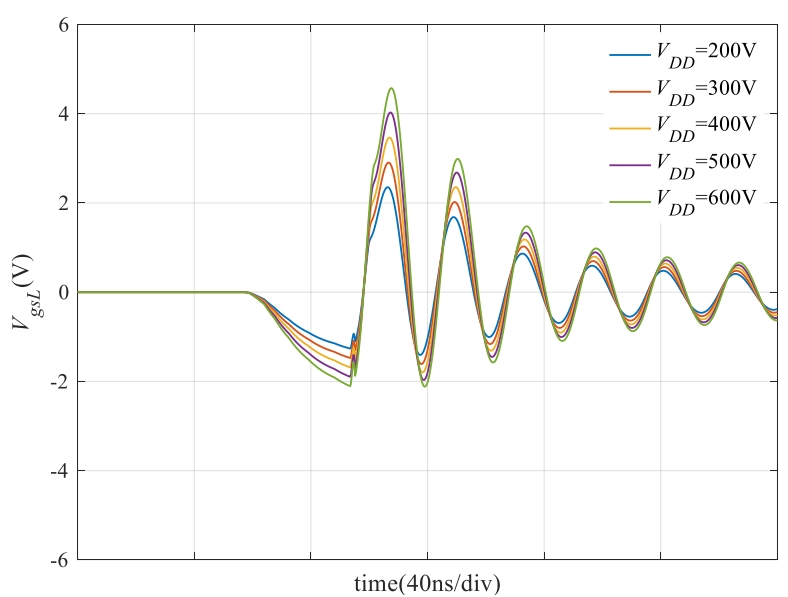

(a)

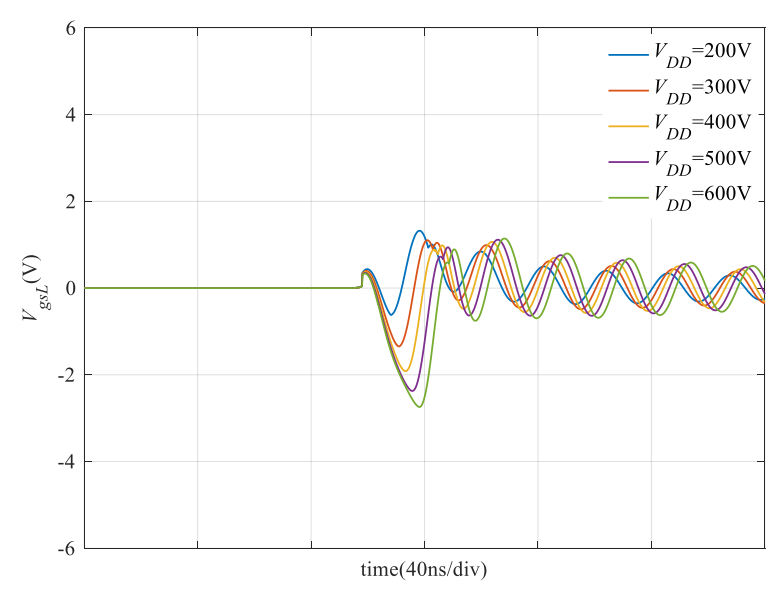

(b)

Figure 23. Cont. 


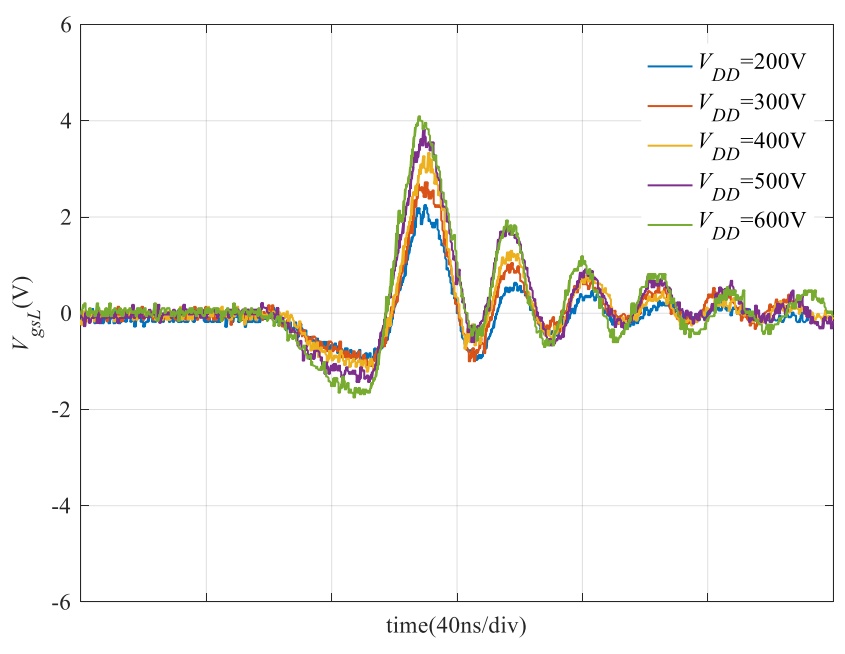

(c)

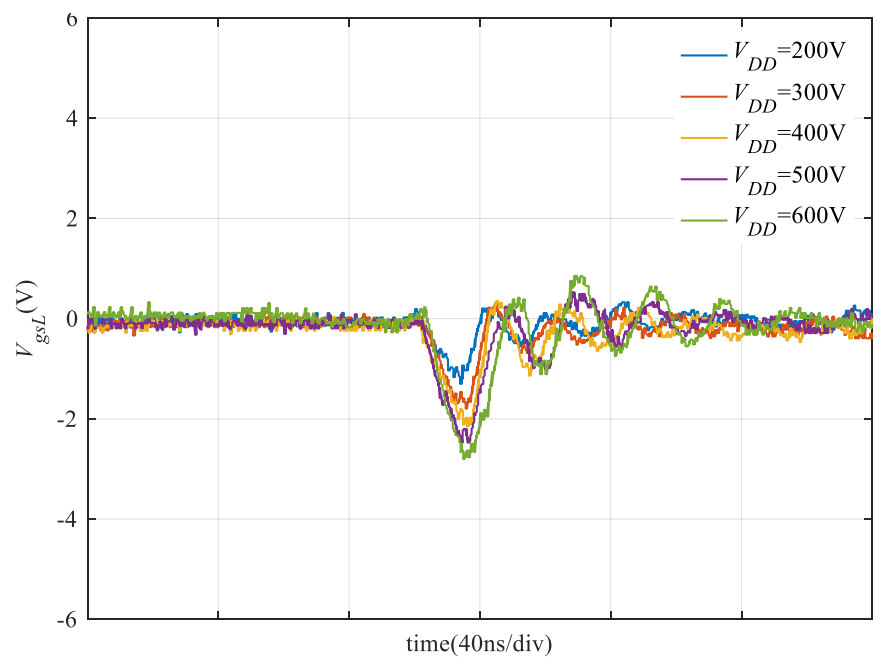

(d)

Figure 23. Comparison of simulation and experimental waveforms of crosstalk with different $V_{D D}$. (a,b): simulation. (c,d): experiment. Left: turn-on. Right: turn-off.

Table 6. Comparison of Experiments and Simulations with Variable $V_{D D}$.

\begin{tabular}{|c|c|c|c|c|c|c|}
\hline & $\mathrm{V}_{\mathrm{DD}}(\mathrm{V})$ & 200 & 300 & 400 & 500 & 600 \\
\hline \multirow{3}{*}{ Turn-on } & Relative $\mathrm{V}_{\mathrm{gsL}}$ with Simulation (V) & 1.860 & 2.656 & 3.326 & 3.870 & 4.282 \\
\hline & $\begin{array}{c}\text { Relative } \mathrm{V}_{\mathrm{gsL}} \text { with } \\
\text { Experiment }(\mathrm{V})\end{array}$ & 1.81 & 2.58 & 3.22 & 3.72 & 4.05 \\
\hline & Difference compared with experiment & $2.76 \%$ & $1.46 \%$ & $3.29 \%$ & $4.03 \%$ & $5.73 \%$ \\
\hline \multirow{3}{*}{ Turn-off } & Relative $\mathrm{V}_{\mathrm{gsL}}$ with Simulation (V) & -0.911 & -1.554 & -2.166 & -2.510 & -2.742 \\
\hline & $\begin{array}{c}\text { Relative } \mathrm{V}_{\mathrm{gsL}} \text { with } \\
\text { Experiment }(\mathrm{V})\end{array}$ & -1.00 & -1.61 & -2.11 & -2.42 & -2.59 \\
\hline & Difference compared with experiment & $8.90 \%$ & $3.48 \%$ & $2.65 \%$ & $3.72 \%$ & $5.87 \%$ \\
\hline
\end{tabular}

As shown in Figure 23, the experimental waveforms of crosstalk agree well with the simulation results with the different supply voltage. Figure 24 shows the comparisons of the relative crosstalk peak values extracted from the experiments with the simulation results and the calculated values according to the algorithm of [24]. It can be seen that the crosstalk peaks simulated by the proposed model are more accurate than the crosstalk peaks values calculated based on the method in [24]. This can be attributed to the errors caused by the parasitic inductances, especially in the turn-off process, the induced voltage on $L_{S L}$ and the impedance of the driver loop have opposite effects on the fluctuation of $V_{g s L}$, which causes greater deviations. 


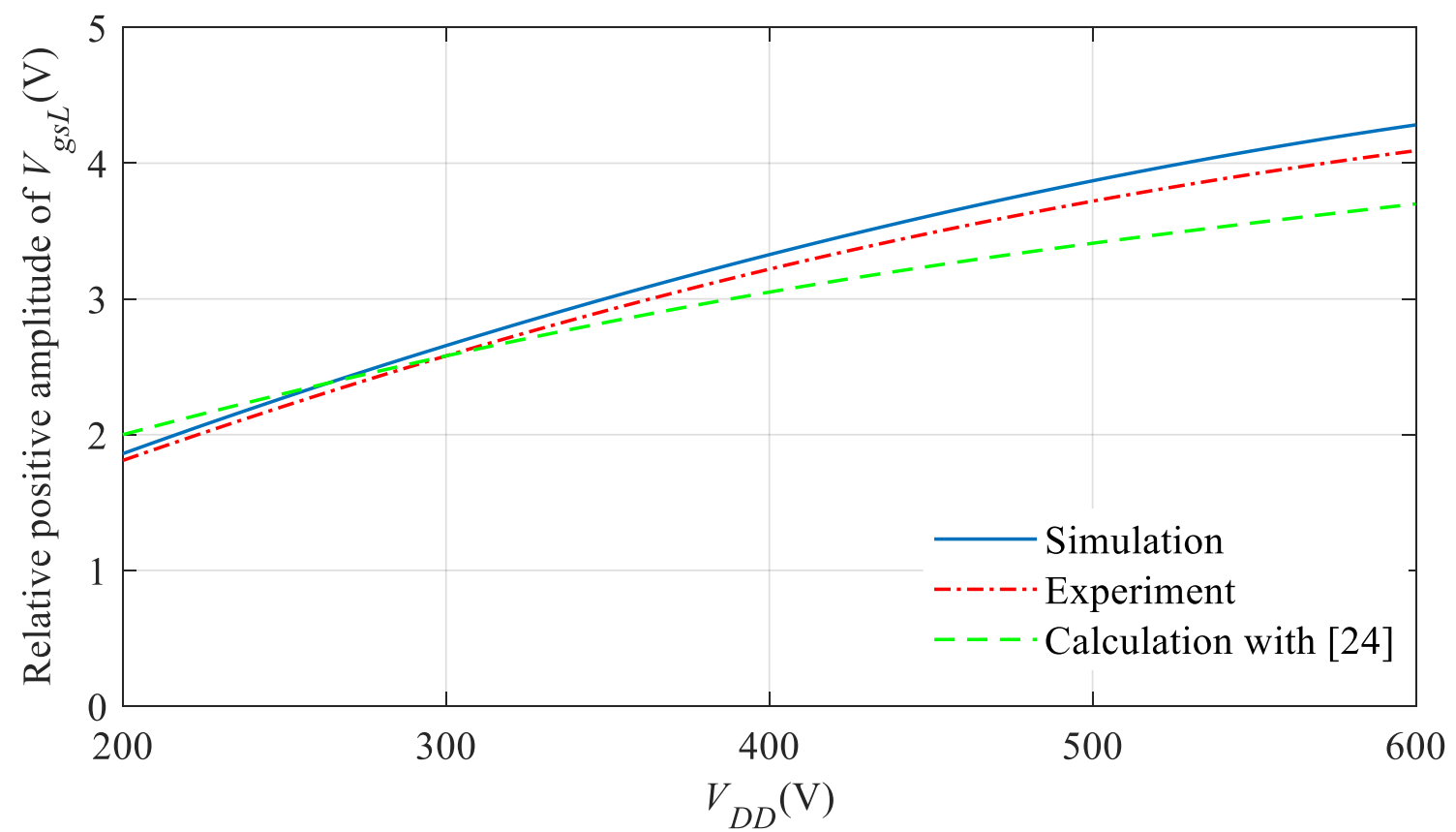

(a)

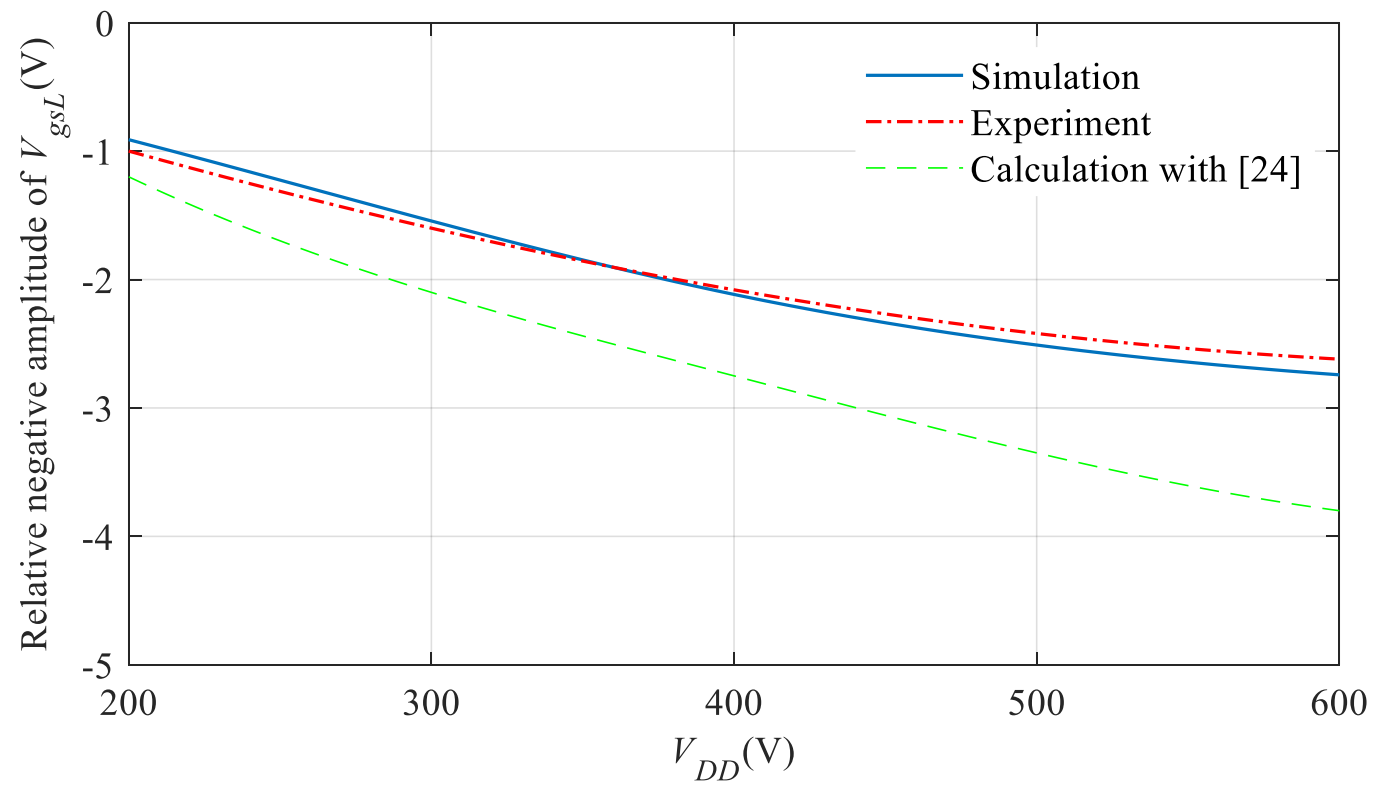

(b)

Figure 24. Comparison of simulation and experimental variation trend of relative crosstalk peak value with different $V_{D D}$. (a) turn-on. (b) turn-off.

\section{Conclusions}

In this paper, a detailed and accurate analytical model of crosstalk for SiC MOSFETs bridge-leg configuration is proposed. The source impact variables and the critical parasitic elements to the crosstalk are comprehensively considered. The critical factors of $\mathrm{SiC}$ MOSFETs and bridge-arm are taken into account, including the nonlinear characteristics of the interelectrode capacitances, the reverse recovery characteristics of the anti-parallel diodes, the parasitic inductances of the power loop, and the nonlinear voltage switching and damping oscillation process. Their direct or indirect coupling effects to the crosstalk in different switching stages are analyzed. Combining with these factors, the proposed model covers the whole crosstalk response of the bridge-arm switching processes and 
the equivalent analysis circuits of each switching stage are established. The correctness and the accuracy of the proposed model are validated by simulation and experiment. Compared with the current crosstalk model, the proposed model can present more details and accurately of crosstalk prediction and is closer to the practical engineering application. The proposed model can be employed to evaluate the crosstalk responses to ensure the stability and reliability of bridge-arms, and can also be used in the design and development of power converters to reduce loss and play full operation performances of SiC MOSFETs. In future work, the influence trends of the parasitic elements and variables on the crosstalk response can be detailed analyzed based on the model. It should be noticed that the temperature characteristics of the model are not concerned in this paper, this may cause deviations between the modeling values and the actual values of parasitic parameters, and in practical projects, the temperature of the switching devices of power converters varies with the operating time. Thus, the improved analytical model for crosstalk with considering temperature characteristics will be studied in future work to improve the accuracy.

Author Contributions: Conceptualization, D.Y. and X.W.; methodology, D.Y.; validation, D.Y. and X.W.; formal analysis, D.Y.; investigation, D.Y. and X.W.; writing—original draft preparation, D.Y.; review and editing, Y.Z. and X.W.; supervision, Y.Z.; project administration, Y.Z. All authors have read and agreed to the published version of the manuscript.

Funding: This work was supported by the Strategic Priority Research Program of the Chinese Academy of Sciences XDA14020400.

Conflicts of Interest: The authors declare no conflict of interest.

\section{References}

1. Liang, Z.; Ning, P.; Wang, F. Development of Advanced All-SiC Power Modules. IEEE Trans. Power Electron. 2014, 29, 2289-2295. [CrossRef]

2. Garrido-Diez, D.; Baraia, I. Review of wide bandgap materials and their impact in new power devices. In Proceedings of the 2017 IEEE International Workshop of Electronics, Control, Measurement, Signals and their Application to Mechatronics (ECMSM), Donostia-San Sebastian, Spain, 24-26 May 2017; pp. 1-6.

3. Zhao, Z.; He, X. Research on Digital Synchronous Rectification for a High-Efficiency DC-DC Converter in an Auxiliary Power Supply System of Magnetic Levitation. Energies 2019, 13, 51. [CrossRef]

4. Biela, J.; Schweizer, M.; Waffler, S.; Kolar, J.W. SiC versus Si-Evaluation of Potentials for Performance Improvement of Inverter and DC-DC Converter Systems by SiC Power Semiconductors. IEEE Trans. Ind. Electron. 2011, 58, 2872-2882. [CrossRef]

5. Wang, Z.; Shi, X.; Xue, Y.; Tolbert, L.M.; Wang, F.; Blalock, B.J. Design and Performance Evaluation of Overcurrent Protection Schemes for Silicon Carbide (SiC) Power MOSFETs. IEEE Trans. Ind. Electron. 2014, 61, 5570-5581. [CrossRef]

6. Funaki, T. A study on self turn-on phenomenon in fast switching operation of high voltage power MOSFET. In Proceedings of the 2013 3rd IEEE CPMT Symposium Japan, Kyoto, Japan, 11-13 November 2013; pp. 1-4.

7. Zaman, H.; Wu, X.; Zheng, X.; Khan, S.; Ali, H. Suppression of Switching Crosstalk and Voltage Oscillations in a SiC MOSFET Based Half-Bridge Converter. Energies 2018, 11, 3111. [CrossRef]

8. Zhang, Z.; Zhang, W.; Wang, F.; Tolbert, L.M.; Blalock, B.J. Analysis of the switching speed limitation of wide band-gap devices in a phase-leg configuration. In Proceedings of the 2012 IEEE Energy Conversion Congress and Exposition (ECCE), Raleigh, NC, USA, 15-20 September 2012; pp. 3950-3955.

9. Chen, Z; Boroyevich, D.; Burgos, R.; Wang, F. Characterization and modeling of $1.2 \mathrm{kv}, 20 \mathrm{~A}$ SiC MOSFETs. In Proceedings of the 2009 IEEE Energy Conversion Congress and Exposition, San Jose, CA, USA, 20-24 September 2009; pp. 1480-1487.

10. Merienne, F.; Roudet, J.; Schanen, J. Switching disturbance due to source inductance for a power MOSFET: Analysis and solutions. In Proceedings of the PESC Record. 27th Annual IEEE Power Electronics Specialists Conference, Baveno, Italy, 23-27 June 2002; Volume 2, pp. 1743-1747.

11. Wang, Q. The Impact of Parasitic Elements on Spurious Turn-On in Phase-Shifted Full-Bridge Converters. J. Power Electron. 2016, 16, 883-893. [CrossRef]

12. Jahdi, S.; Alatise, O.; Alexakis, P.; Ran, L.; Mawby, P. The Impact of Temperature and Switching Rate on the Dynamic Characteristics of Silicon Carbide Schottky Barrier Diodes and MOSFETs. IEEE Trans. Ind. Electron. 2015, 62, 163-171. [CrossRef]

13. Yin, S.; Tseng, K.J.; Tong, C.F.; Simanjorang, R.; Gajanayake, C.J.; Nawawi, A.; Liu, Y.; Liu, Y.; See, K.Y.; Sakanova, A.; et al. Gate driver optimization to mitigate shoot-through in high-speed switching SiC half bridge module. In Proceedings of the 2015 IEEE 11th International Conference on Power Electronics and Drive Systems, Sydney, NSW, Australia, 9-12 June 2015; pp. 484-491.

14. Wang, P.; Zhang, L.; Lu, X.; Sun, H.; Wang, W.; Xu, D. An Improved Active Crosstalk Suppression Method for High-Speed SiC MOSFETs. IEEE Trans. Ind. Appl. 2019, 55, 7736-7744. [CrossRef] 
15. Elbanhawy, A. Limiting Cross-Conduction Current in Synchronous Buck Converter Designs; Note AN-7019; Fairchild Semiconductor: San Jose, CA, USA, 2005.

16. Zhou, Q.; Gao, F. A gate driver of SiC MOSFET for suppressing the negative voltage spikes in a bridge circuit. In Proceedings of the 2016 IEEE Applied Power Electronics Conference and Exposition (APEC), Long Beach, CA, USA, 20-24 March 2016; pp. 536-543.

17. $\mathrm{Wu}, \mathrm{T} . \mathrm{Cdv} / \mathrm{dt}$ induced turn-on in synchronous buck regulators. Proc. Appl. Manual-Int. Rectifier 2012, 1-6.

18. Li, Y.; Liang, M.; Chen, J.; Zheng, T.Q.; Guo, H. A Low Gate Turn-OFF Impedance Driver for Suppressing Crosstalk of SiC MOSFET Based on Different Discrete Packages. IEEE J. Emerg. Sel. Top. Power Electron. 2019, 7, 353-365. [CrossRef]

19. Nishigaki, A.; Umegami, H.; Hattori, F.; Martinez, W.; Yamamoto, M. An analysis of false turn-on mechanism on power devices. In Proceedings of the 2014 IEEE Energy Conversion Congress and Exposition (ECCE), Pittsburgh, PA, USA, 14-18 September 2014; pp. 2988-2993.

20. Jahdi, S.; Alatise, O.; Gonzalez, J.A.O.; Bonyadi, R.; Ran, L.; Mawby, P. Temperature and Switching Rate Dependence of Crosstalk in Si-IGBT and $\mathrm{SiC}$ Power Modules. IEEE Trans. Ind. Electron. 2016, 63, 849-863. [CrossRef]

21. Wang, J.; Chung, H.S.-H. Impact of Parasitic Elements on the Spurious Triggering Pulse in Synchronous Buck Converter. IEEE Trans. Power Electron. 2014, 29, 6672-6685. [CrossRef]

22. Elferich, R.; López, T. Impact of Gate Voltage Bias on Reverse Recovery Losses of Power MOSFETs. In Proceedings of the Twenty-First Annual IEEE Applied Power Electronics Conference and Exposition, APEC '06, Dallas, TX, USA, 19-23 March 2006; p. 6.

23. Zhang, B.; Xie, S.; Xu, J.; Qian, Q.; Zhang, Z.; Xu, K. A Magnetic Coupling Based Gate Driver for Crosstalk Suppression of SiC MOSFETs. IEEE Trans. Ind. Electron. 2017, 64, 9052-9063. [CrossRef]

24. Li, H.; Jiang, Y.; Qiu, Z.; Wang, Y.; Ding, Y. A Predictive Algorithm for Crosstalk Peaks of SiC MOSFET by Considering the Nonlinearity of Gate-Drain Capacitance. IEEE Trans. Power Electron. 2021, 36, 2823-2834. [CrossRef]

25. Mudholkar, M.; Ahmed, S.; Ericson, M.N.; Frank, S.S.; Britton, C.L.; Mantooth, H.A. Datasheet Driven Silicon Carbide Power MOSFET Model. IEEE Trans. Power Electron. 2013, 29, 2220-2228. [CrossRef]

26. Costinett, D.; Maksimovic, D.; Zane, R. Circuit-Oriented Treatment of Nonlinear Capacitances in Switched-Mode Power Supplies. IEEE Trans. Power Electron. 2015, 30, 985-995. [CrossRef]

27. Chen, K.; Zhao, Z.; Yuan, L.; Lu, T.; He, F. The Impact of Nonlinear Junction Capacitance on Switching Transient and Its Modeling for SiC MOSFET. IEEE Trans. Electron Devices 2015, 62, 333-338. [CrossRef]

28. Duan, Z.; Fan, T.; Wen, X.; Zhang, D. Improved SiC Power MOSFET Model Considering Nonlinear Junction Capacitances. IEEE Trans. Power Electron. 2018, 33, 2509-2517. [CrossRef]

29. Ho, C.N.M.; Canales, F.; Coccia, A.; Laitinen, M. A Circuit-Level Analytical Study on Switching Behaviors of SiC Diode at Basic Cell for Power Converters. In Proceedings of the 2008 IEEE Industry Applications Society Annual Meeting, Edmonton, AB, Canada, 5-9 October 2008; pp. 1-8.

30. Chen, Z.; Amaro, I. Optimizing low side gate resistance for damping phase node ringing of synchronous buck converter. In Proceedings of the 2012 IEEE Energy Conversion Congress and Exposition (ECCE), Raleigh, NC, USA, 15-20 September 2012; pp. 1827-1832.

31. Xue, P.; Maresca, L.; Riccio, M.; Breglio, G.; Irace, A. Analysis on the Self-Sustained Oscillation of SiC MOSFET Body Diode. IEEE Trans. Electron Devices 2019, 66, 4287-4295. [CrossRef]

32. Bi, C.; Lu, R.; Li, H. Prediction of Electromagnetic Interference Noise in SiC MOSFET Module. IEEE Trans. Circuits Syst. II Express Briefs 2019, 66, 853-857. [CrossRef]

33. Yang, J.; Feng, G.; Huadong, L.; Suhong, C. Characterization and Detailed Analysis of the Crosstalk with SiC MOSFET. In Proceedings of the 2018 1st Workshop on Wide Bandgap Power Devices and Applications in Asia (WiPDA Asia), Xi'an, China, 16-18 May 2018; pp. 239-243. [CrossRef]

34. Wang, J.; Chung, H.S.-H.; Li, R.T.-H. Characterization and Experimental Assessment of the Effects of Parasitic Elements on the MOSFET Switching Performance. IEEE Trans. Power Electron. 2013, 28, 573-590. [CrossRef]

35. Liang, M.; Zheng, T.Q.; Li, Y. An Improved Analytical Model for Predicting the Switching Performance of SiC MOSFETs. J. Power Electron. 2016, 16, 374-387. [CrossRef]

36. Christen, D.; Biela, J. Analytical Switching Loss Modeling Based on Datasheet Parameters for mosfets in a Half-Bridge. IEEE Trans. Power Electron. 2019, 34, 3700-3710. [CrossRef]

37. Li, X.; Jiang, J.; Huang, A.Q.; Guo, S.; Deng, X.; Zhang, B.; She, X. A SiC Power MOSFET Loss Model Suitable for High-Frequency Applications. IEEE Trans. Ind. Electron. 2017, 64, 8268-8276. [CrossRef]

38. Lauritzen, P.; Ma, C. A simple diode model with reverse recovery. IEEE Trans. Power Electron. 1991, 6, 188-191. [CrossRef]

39. Clarks, K.A.; Matthew, N.O.S. Fundamentals of Electric Circuits, 5th ed.; McGraw-Hill: New York, NY, USA, 2013 ; pp. 52-58.

40. Liu, Z.; Huang, X.; Lee, F.C.; Li, Q. Package Parasitic Inductance Extraction and Simulation Model Development for the High-Voltage Cascode GaN HEMT. IEEE Trans. Power Electron. 2014, 29, 1977-1985. [CrossRef] 